\title{
Revisiting the wrinkling of elastic bilayers II: Post-bifurcation analysis
}

\author{
Hamza Alawiye*, Patrick E. Farrell*, Alain Goriely* \\ Mathematical Institute, University of Oxford, Oxford OX2 6GG, UK
}

\begin{abstract}
We examine the problem of an elastic film bonded to an elastic substrate and subjected to compressive stress (induced by growth of the film or lateral compression of the whole system). Following the linear analysis previously carried out to determine the critical growth/compression ratio required to induce wrinkling in the film, we carry out a weakly-nonlinear analysis to derive an amplitude equation that describes the evolution of the wrinkling amplitude beyond the bifurcation point. We carry out a comprehensive numerical bifurcation analysis of the problem using the finite element method and show excellent agreements between the weakly-nonlinear analysis and the numerical experiments. We are also able to solve directly for the bifurcation point in our discretized system and characterize the effect of implementation details such as the aspect ratio of the computational domain on the observed bifurcation point. Finally, we explore solutions of the amplitude equation in the case that the wrinkling amplitude is allowed to vary over long spatial and/or temporal scales.
\end{abstract}

Keywords: buckling, nonlinear elasticity, asymptotic analysis, finite elements, stability and bifurcation

2010 MSC: 74B20

\section{Introduction}

Understanding the post-buckling behavior of structures in solid mechanics is a problem of central importance. Failure modes such as plastic deformation and fracture vastly complicate the mathematical analysis of these problems in engineering contexts, but even when these added phenomena are cast aside, one is still left with the task of understanding the solution set of a highly nonlinear partial differential equation. Despite its inherent complexity, this field of study has continued to find new applications - especially in the study of soft materials, where buckling is not necessarily associated with failure. In particular, it is relevant in understanding situations involving growing biological materials [30] such as the morphogenesis of human brains [6, 31], intestines [7, skin [20] and other organs [19, 45]; but also in traditional engineering contexts for the measurement of material properties [17], stimuli-responsive biomaterial design [41, 14, flexible electronics [40, 42] and optics [9].

One such structure that has gathered particular interest is the elastic bilayer: a thin, elastic film bonded to the top of a large, elastic substrate. It is well known, both experimentally and analytically,

${ }^{*}$ Corresponding Author

Email address: alawiye@maths.ox.ac.uk (Hamza Alawiye) 
that if the film is significantly stiffer than the substrate, sufficient compression or growth induced strain will cause the formation of a periodic wrinkling pattern to emerge.

A number of previous works have examined various formulations of this problem from different perspectives. Cai and Fu [13] offered one of the first in-depth treatments of the fully nonlinear elastic problem where both the film and substrate are modeled using neo-Hookean elasticity (or another hyperelastic constitutive relation). Subsequent works have examined substrate pre-stretch [15, 38, systems with smoothly varying stiffness [24, 23], systems with small stiffness contrasts [28], a secondary period-doubling bifurcation [26], strain-gradient materials [21], different compression modes [36] and three-dimensional pattern selection [16].

In part I of this work [1], we presented a unified framework based on the theory of morphoelasticity [30] to understand combined growth-compression problems and determining the critical growth and/or compression ratio threshold required for the formation of such wrinkling patterns in the presence of a variety of physical phenomena. In this work, we extend this analysis to the post-buckling regime using weakly-nonlinear analysis and provide numerical verification of its results.

We first briefly review the linear analysis presented in Alawiye et al. [1] before performing higher order perturbation analysis including a mode amplitude that can vary over slow spatial and temporal scales. At third order, we find a compatibility condition or amplitude relation that describes the evolution of the mode amplitude past the critical bifurcation parameter. We then detail a procedure for a finite element solution of our problem along with a numerical bifurcation analysis scheme to discover systematically and enumerate the nontrivial solution branches.

\section{The model}

\subsection{General formulation}
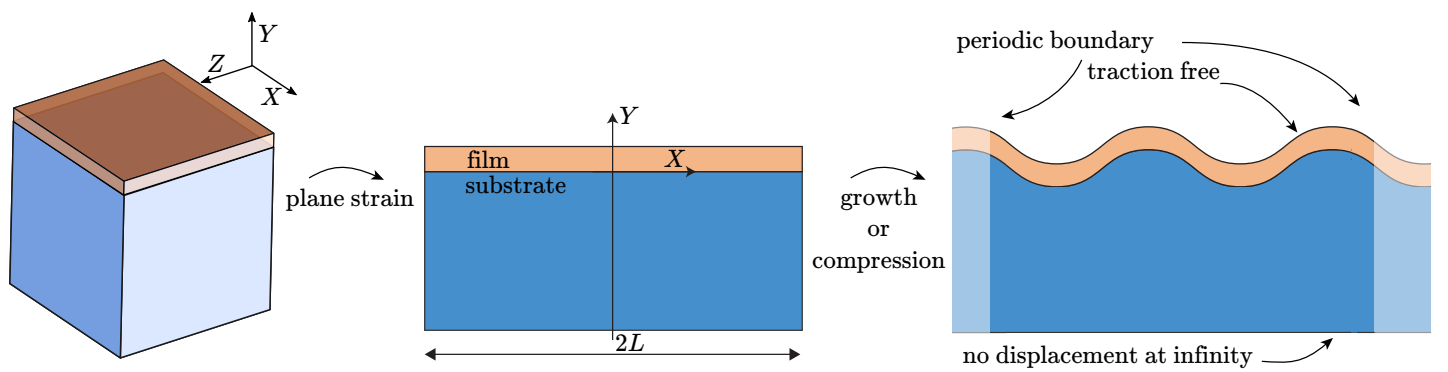

Figure 1: Geometry of the domain. The system is composed of a bilayer with an infinitely deep layer of width $2 L$ bonded by a film of thickness 1 . Considering only plane strain, the problem is reduced to the deformation of a two-dimensional system under either compression or growth causing wrinkling. The boundary conditions are: continuity of traction and displacement between the layers, sliding vertical boundaries with no detachment, traction free upper layer, and no displacement or detachment at infinity.

We model a morphoelastic bilayer (illustrated in Figure 1) as a growing incompressible neo-Hookean film of unit thickness (denoted $\mathcal{B}_{\mathrm{f}}$ ) attached to a semi-infinite incompressible neo-Hookean substrate (denoted $\mathcal{B}_{\mathrm{s}}$ ). We denote the ratio of the shear moduli of the two layers by $\beta:=\mu_{\mathrm{f}} / \mu_{\mathrm{s}}$. In Alawiye et al. [1, we performed a linear analysis of this system under either growth with a multiplicative volume factor of $J:=g^{2}$ given by

$$
g(\mathbf{X}):= \begin{cases}\gamma & \mathbf{X} \in \mathcal{B}_{\mathrm{f}} \\ 1 & \mathbf{X} \in \mathcal{B}_{\mathrm{s}}\end{cases}
$$


or lateral compression of the whole bilayer system with compression ratio $\lambda$. In particular, we detailed how we can impose the elastic incompressibility condition exactly with a stream function construction, thus avoiding the use of Lagrange multipliers. The stream function $\Psi$ operates on a mixture of the reference coordinates $(X, Y)$ and the deformed coordinates $(x, y)$. The theory is equivalent for either choice of mixed coordinates, but it is mathematically convenient to use $(x, Y)$ for the particular geometry in question. We refer to this article for the details of the construction but we recall that the deformation gradient can be written in terms of a stream function $\Psi(x, Y)$ as follows:

$$
\mathbf{F}=\frac{1}{\partial_{x Y} \Psi}\left(\begin{array}{cc}
J \lambda & -\partial_{Y Y} \Psi \\
J \partial_{x x} \Psi & \lambda^{-1}\left(\left(\partial_{x Y} \Psi\right)^{2}-\partial_{Y Y} \Psi \partial_{x x} \Psi\right)
\end{array}\right)
$$

Substituting this into the neo-Hookean energy density

$$
W(\mathbf{A})=\frac{\mu}{2} \operatorname{tr}\left(\mathbf{A} \mathbf{A}^{T}-\mathbf{I}\right), \quad \text { where } \mathbf{A}=\mathbf{F}\left(\begin{array}{cc}
\lambda^{-1} g^{-1} & 0 \\
0 & \lambda g^{-1}
\end{array}\right)
$$

yields a minimization problem of the following form for each layer:

$$
\begin{aligned}
\underset{\Psi \in \Phi}{\operatorname{minimize}} \tilde{I}(\Psi):=\int_{\tilde{\mathcal{B}}} \frac{\mu}{2 J \lambda^{3} \partial_{x Y} \Psi}[ & -2 J \lambda^{2}\left(\partial_{x Y} \Psi\right)^{2}+\left(\partial_{x Y} \Psi\right)^{4}-2 \partial_{Y Y} \Psi\left(\partial_{x Y} \Psi\right)^{2} \partial_{x x} \Psi+ \\
& \left.+J^{2} \lambda^{2}\left(\lambda^{2}+\left(\partial_{x x} \Psi\right)^{2}\right)+\left(\partial_{Y Y} \Psi\right)^{2}\left(\lambda^{2}+\left(\partial_{x x} \Psi\right)^{2}\right)\right] \mathrm{d} x \mathrm{~d} Y,
\end{aligned}
$$

where $\boldsymbol{\Phi}$ is the set of admissible stream functions, $\tilde{\mathcal{B}}$ is the domain in mixed coordinates and $\mu$ is the shear modulus. We can then compute the Euler-Lagrange equations corresponding to the minimization problems:

$$
\begin{aligned}
& \frac{\partial^{2}}{\partial x^{2}}\left(\frac{\partial f}{\partial\left(\partial_{x x} \Psi_{\mathrm{f}}\right)}\right)+\frac{\partial^{2}}{\partial x \partial Y}\left(\frac{\partial f}{\partial\left(\partial_{x Y} \Psi_{\mathrm{f}}\right)}\right)+\frac{\partial^{2}}{\partial Y^{2}}\left(\frac{\partial f}{\partial\left(\partial_{Y Y} \Psi_{\mathrm{f}}\right)}\right)=0 \\
& \frac{\partial^{2}}{\partial x^{2}}\left(\frac{\partial f}{\partial\left(\partial_{x x} \Psi_{\mathrm{s}}\right)}\right)+\frac{\partial^{2}}{\partial x \partial Y}\left(\frac{\partial f}{\partial\left(\partial_{x Y} \Psi_{\mathrm{s}}\right)}\right)+\frac{\partial^{2}}{\partial Y^{2}}\left(\frac{\partial f}{\partial\left(\partial_{Y Y} \Psi_{\mathrm{s}}\right)}\right)=0 .
\end{aligned}
$$

After perturbation expansions $\Psi=\Psi^{(0)}+\epsilon \Psi^{(1)}$ around the trivial solution $\Psi^{(0)}(x, Y)=J x Y$ and periodic decompositions $\Psi^{(1)}(x, Y)=\sin (k x) h^{(1)}(Y)$, we arrive at a system of two fourth order, linear ODE coupled through their boundary conditions (given in Appendix A). In the growth case, the application of these boundary conditions yields a solubility condition of the form

$$
\mathbf{M}(k, \gamma, \beta) \mathbf{c}=\mathbf{0},
$$

where our system only has non-trivial solutions if $\operatorname{det} \mathbf{M}=0$. Asymptotic or numerical solution of this condition yielded critical values $k_{\mathrm{cr}}$ and $\gamma_{\mathrm{cr}}$ for the wavenumber and growth factor respectively as functions of $\beta$ (shown in Figure 2). A condition of the same form was computed for the compression case, yielding a critical compression ratio $\lambda_{\text {cr }}$. Note that the amplitude of the wrinkling solutions is left undetermined at this point. 


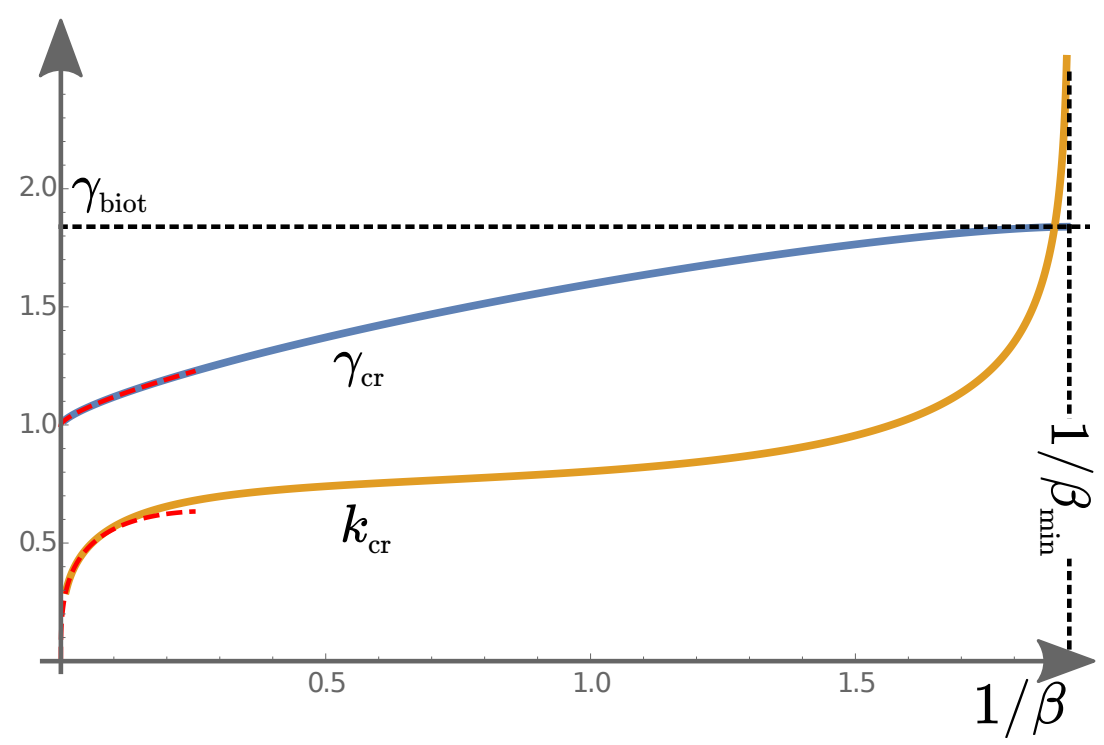

Figure 2: Critical value of growth and wavenumber as a function of $\beta^{-1}$ (asymptotic approximations shown dashed). Critical solutions exist for $\beta>\beta_{\min } \approx 1.9^{-1}$ after which, the Biot instability is the dominant instability.

\section{Derivation of the amplitude relation}

\subsection{Growth case}

We first focus on the growth case and extend our previous analysis by including higher order terms in our perturbation expansion of the stream function. This will allow us to derive a so-called amplitude equation, the solutions of which specify the as-of-yet undetermined amplitude of the oscillatory solutions. At this point, we also allow the amplitude to depend on a far spatial $\zeta:=\epsilon x$ and a slow temporal variable $\tau:=\epsilon t$ that evolve over longer length and time scales than the oscillations themselves. We consider perturbations of the form

$$
\Psi=\Psi^{(0)}+\epsilon \Psi^{(1)}+\epsilon^{2} \Psi^{(2)}+\epsilon^{3} \Psi^{(3)},
$$

and furthermore perturb the critical parameter

$$
\gamma=\gamma_{\mathrm{cr}}\left(1+\epsilon^{2} \gamma_{1}\right)
$$

We then take periodic decompositions of the form

$$
\begin{aligned}
\Psi^{(1)}(x, Y, t)= & a(\epsilon x, \epsilon t) h^{(1,1)}(Y) e^{i k x}+\text { c.c. } \\
\Psi^{(2)}(x, Y, t)= & a(\epsilon x, \epsilon t)^{2} h^{(2,2)}(Y) e^{2 i k x}+\partial_{\zeta} a(\epsilon x, \epsilon t) h^{(2,1)}(Y) e^{i k x}+\text { c.c. } \\
\Psi^{(3)}(x, Y, t)= & a(\epsilon x, \epsilon t)^{3} h^{(3,3)}(Y) e^{3 i k x}+2 a(\epsilon x, \epsilon t) \partial_{\zeta} a(\epsilon x, \epsilon t) h^{(3,2)}(Y) e^{2 i k x} \\
& +\partial_{\zeta \zeta} a(\epsilon x, \epsilon t) h^{(3,1)}(Y) e^{i k x}+\text { c.c. },
\end{aligned}
$$

where c.c. denotes the complex conjugate of all preceding expressions. To deal with the added slow dynamics of the system, we replace our neo-Hookean energy density $W$ with the Lagrangian density

$$
\frac{1}{2} \rho\left|\frac{\partial \mathbf{x}}{\partial t}\right|^{2}-W(\mathbf{A}) .
$$


To use this in our formulation, we must be able to express the kinetic energy of the system in terms of stream functions. We can compute the components of the time derivative of the displacement as

$$
\begin{aligned}
& \frac{\partial x}{\partial t}=-\frac{\partial_{Y t} \Psi}{\partial_{x Y} \Psi} \\
& \frac{\partial y}{\partial t}=\frac{1}{\lambda}\left(\partial_{x t} \Psi-\frac{\partial_{Y t} \Psi \partial_{x x} \Psi}{\partial_{x Y} \Psi}\right) .
\end{aligned}
$$

Combining these expressions with (3.4), we can write the Lagrangian of the system in the form

$$
\tilde{I}(\Psi)=\int_{\tilde{\mathcal{B}}} f\left(\partial_{x x} \Psi, \partial_{x Y} \Psi, \partial_{Y Y} \Psi, \partial_{x t} \Psi, \partial_{Y t} \Psi\right) \mathrm{d} x \mathrm{~d} Y .
$$

The Euler-Lagrange equations are then given by

$$
\begin{aligned}
\frac{\partial^{2}}{\partial x^{2}}\left(\frac{\partial f}{\partial\left(\partial_{x x} \Psi_{\mathrm{f}}\right)}\right)+\frac{\partial^{2}}{\partial x \partial Y}\left(\frac{\partial f}{\partial\left(\partial_{x Y} \Psi_{\mathrm{f}}\right)}\right) & +\frac{\partial^{2}}{\partial Y^{2}}\left(\frac{\partial f}{\partial\left(\partial_{Y Y} \Psi_{\mathrm{f}}\right)}\right) \\
& +\frac{\partial^{2}}{\partial x \partial t}\left(\frac{\partial f}{\partial\left(\partial_{x t} \Psi_{\mathrm{f}}\right)}\right)+\frac{\partial^{2}}{\partial Y \partial t}\left(\frac{\partial f}{\partial\left(\partial_{Y t} \Psi_{\mathrm{f}}\right)}\right)=0 \\
\frac{\partial^{2}}{\partial x^{2}}\left(\frac{\partial f}{\partial\left(\partial_{x x} \Psi_{\mathrm{s}}\right)}\right)+\frac{\partial^{2}}{\partial x \partial Y}\left(\frac{\partial f}{\partial\left(\partial_{x Y} \Psi_{\mathrm{s}}\right)}\right) & +\frac{\partial^{2}}{\partial Y^{2}}\left(\frac{\partial f}{\partial\left(\partial_{Y Y} \Psi_{\mathrm{s}}\right)}\right) \\
& +\frac{\partial^{2}}{\partial x \partial t}\left(\frac{\partial f}{\partial\left(\partial_{x t} \Psi_{\mathrm{s}}\right)}\right)+\frac{\partial^{2}}{\partial Y \partial t}\left(\frac{\partial f}{\partial\left(\partial_{Y t} \Psi_{\mathrm{s}}\right)}\right)=0
\end{aligned}
$$

The linear analysis we have already carried out is unchanged by these modifications (although we relabel $h_{\mathrm{f}}^{(1)}$ as $h_{\mathrm{f}}^{(1,1)}$ and $c_{i}$ as $c_{i}^{(1,1)}$ ), so we proceed to the second order. After substituting (3.1) into (2.5), the Euler-Lagrange equations for the first mode at second order in $\epsilon$ read

$$
\begin{aligned}
& \frac{\mathrm{d}^{4} h_{\mathrm{f}}^{(2,1)}}{\mathrm{d} Y^{4}}-k^{2}\left(1+\gamma_{\mathrm{cr}}^{4}\right) \frac{\mathrm{d}^{2} h_{\mathrm{f}}^{(2,1)}}{\mathrm{d} Y^{2}}+\gamma_{\mathrm{cr}}^{4} k^{4} h_{\mathrm{f}}^{(2,1)}=-2 i k\left(1+\gamma_{\mathrm{cr}}^{4}\right) \frac{\mathrm{d}^{2} h_{\mathrm{f}}^{(1,1)}}{\mathrm{d} Y^{2}}+4 i k^{3} \gamma_{\mathrm{cr}}^{4} h_{\mathrm{f}}^{(1,1)}, \\
& \frac{\mathrm{d}^{4} h_{\mathrm{s}}^{(2,1)}}{\mathrm{d} Y^{4}}-2 k^{2} \frac{\mathrm{d}^{2} h_{\mathrm{s}}^{(2,1)}}{\mathrm{d} Y^{2}}+k^{4} h_{\mathrm{s}}^{(2,1)}=-4 i k \frac{\mathrm{d}^{2} h_{\mathrm{s}}^{(1,1)}}{\mathrm{d} Y^{2}}+4 i k^{3} h_{\mathrm{s}}^{(1,1)} .
\end{aligned}
$$

We also obtain a complex conjugate set of equations. On substitution of the first order solution, we can write each of the equations (3.8) in the form

$$
\mathcal{L}^{(1)}\left[h^{(2,1)}\right]=\mathscr{F}^{(2,1)},
$$

where $\mathcal{L}^{(n)}$ is one of the linear differential operators

$$
\begin{aligned}
\mathcal{L}_{\mathrm{f}}^{(n)}[h]:= & \frac{\mathrm{d}^{4} h}{\mathrm{~d} Y^{4}}-(n k)^{2}\left(1+\gamma_{\mathrm{cr}}^{4}\right) \frac{\mathrm{d}^{2} h}{\mathrm{~d} Y^{2}}+\gamma_{\mathrm{cr}}^{4}(n k)^{4} h, \\
& \mathcal{L}_{\mathrm{s}}^{(n)}[h]:=\frac{\mathrm{d}^{4} h}{\mathrm{~d} Y^{4}}-2(n k)^{2} \frac{\mathrm{d}^{2} h}{\mathrm{~d} Y^{2}}+(n k)^{4} h,
\end{aligned}
$$

and $\mathscr{F}^{(2,1)}$ represents a (known) scalar, nonlinear function of $\beta$ (through $\gamma_{\text {cr }}$ and $k$ ). The equations 
for the second mode at second order can also be written in a similar form:

$$
\mathcal{L}^{(2)}\left[h^{(2,2)}\right]=\mathscr{F}^{(2,2)} .
$$

We can then solve these equations exactly using computer-based symbolic algebra as the particular integrals are straightforward but intractable by hand. By again considering decay conditions of the form $\lim _{Y \rightarrow-\infty} h_{\mathrm{S}}=0$, we obtain the general solutions

$$
\begin{aligned}
& h_{\mathrm{f}}^{(2,1)}(Y)=c_{1}^{(2,1)} e^{-k \gamma_{\mathrm{cr}}^{2} Y}+c_{2}^{(2,1)} e^{k \gamma_{\mathrm{cr}}^{2} Y}+c_{3}^{(2,1)} e^{-k Y}+c_{4}^{(2,1)} e^{k Y}+\mathscr{G}_{\mathrm{f}}^{(2,1)}, \\
& h_{\mathrm{s}}^{(2,1)}(Y)=c_{5}^{(2,1)} e^{k Y}+c_{6}^{(2,1)} Y e^{k Y}+\mathscr{G}_{\mathrm{s}}^{(2,1)}
\end{aligned}
$$

and

$$
\begin{aligned}
& h_{\mathrm{f}}^{(2,2)}(Y)=c_{1}^{(2,2)} e^{-2 k \gamma_{\mathrm{cr}}^{2} Y}+c_{2}^{(2,2)} e^{2 k \gamma_{\mathrm{cr}}^{2} Y}+c_{3}^{(2,2)} e^{-2 k Y}+c_{4}^{(2,2)} e^{2 k Y}+\mathscr{G}_{\mathrm{f}}^{(2,2)}, \\
& h_{\mathrm{s}}^{(2,2)}(Y)=c_{5}^{(2,2)} e^{2 k Y}+c_{6}^{(2,2)} Y e^{2 k Y}+\mathscr{G}_{\mathrm{s}}^{(2,2)},
\end{aligned}
$$

where each $\mathscr{G}$ is a (known) scalar, nonlinear function of $k(\beta)$ and $\gamma_{\mathrm{cr}}(\beta)$. To determine the arbitrary coefficients in $(3.12)$ and $(3.13)$, we must apply the boundary conditions (we omit their precise form for brevity) to obtain two inhomogeneous systems of linear equations of the form

$$
\begin{aligned}
& \mathbf{M}^{(2,1)} \mathbf{c}^{(2,1)}=\mathscr{H}^{(2,1)}, \\
& \mathbf{M}^{(2,2)} \mathbf{c}^{(2,2)}=\mathscr{H}^{(2,2)},
\end{aligned}
$$

for some (known) nonlinear, vector-valued functions $\mathscr{H}$ of $k(\beta), \gamma_{\mathrm{cr}}(\beta)$ and $\beta$. On closer inspection, we find that $\mathbf{M}^{(2,1)}$ is singular and so we must establish whether any solutions to (3.14) do in fact exist. The Fredholm alternative tells us that the system has a solution if and only if every $\boldsymbol{\xi}$ in the left null space of $\mathbf{M}^{(2,1)}$ satisfies

$$
\boldsymbol{\xi} \mathscr{H}^{(2,1)}=0
$$

This condition is satisfied identically and we can proceed to third order.

After substituting (3.1) and the computed first and second-order solutions into (2.5), the EulerLagrange equations for the first mode at third order in $\epsilon$ can be written in a similar form to (3.9):

$$
\mathcal{L}^{(1)}\left[h^{(3,1)}\right]=\mathscr{F}^{(3,1)} .
$$

We finally see the impact of the perturbed growth factor in 3.2 as $\mathscr{F}^{(3,1)}$ is a function of $\gamma_{1}$ in addition to $\gamma_{\mathrm{cr}}(\beta)$ and $k(\beta)$. We then obtain the general solutions

$$
\begin{aligned}
& h_{\mathrm{f}}^{(3,1)}(Y)=c_{1}^{(3,1)} e^{-k \gamma_{\mathrm{cr}}^{2} Y}+c_{2}^{(3,1)} e^{k \gamma_{\mathrm{cr}}^{2} Y}+c_{3}^{(3,1)} e^{-k Y}+c_{4}^{(3,1)} e^{k Y}+\mathscr{G}_{\mathrm{f}}^{(3,1)}, \\
& h_{\mathrm{s}}^{(3,1)}(Y)=c_{5}^{(3,1)} e^{k Y}+c_{6}^{(3,1)} Y e^{k Y}+\mathscr{G}_{\mathrm{s}}^{(3,1)},
\end{aligned}
$$

with $\mathscr{G}_{\mathrm{f}}^{(3,1)}$ featuring dependence on $\gamma_{1}$ in addition to $k(\beta)$ and $\gamma_{\mathrm{cr}}(\beta)$. On applying the boundary conditions, we find a mismatch that will allow us to constrain the amplitude of the oscillations of our solution. The inhomogeneous system of linear equations for the arbitrary coefficients in (3.18) is of the form

$$
\mathbf{M}^{(3,1)} \mathbf{c}^{(3,1)}=\mathscr{H}^{(3,1)},
$$

where $\mathscr{H}^{(3,1)}$ contains the $\gamma_{1}$ dependence. On left-multiplying both sides of 3.19 by a vector $\boldsymbol{\xi}$ from 
the left nullspace of the singular matrix $\mathbf{M}^{(3,1)}$, we obtain

$$
\boldsymbol{\xi} \mathscr{H}^{(3,1)}=0 .
$$

Closer inspection reveals that 3.20$)$ is an equation of the form

$$
\partial_{\tau \tau} a-\mathscr{C}_{2} \partial_{\zeta \zeta} a=\mathscr{C}_{0} \gamma_{1} a+\mathscr{C}_{1}|a|^{2} a,
$$

where $\mathscr{C}_{0}, \mathscr{C}_{1}$ and $\mathscr{C}_{2}$ are real-valued functions of $\beta$. This equation is a nonlinear Klein-Gordon equation, typical for pitchfork bifurcations in spatially-extended conservative problems found in solid mechanics (see e.g. [43, 32]).

For instance, substituting in the previously computed critical parameter values for $\beta=10$ yields $\mathscr{C}_{0} \approx 4.75$ and $\mathscr{C}_{1} \approx-0.05$. In the case of constant amplitude, nontrivial solutions for $a$ are given by

$$
|a|^{2}=-\frac{\mathscr{C}_{0}}{\mathscr{C}_{1}} \gamma_{1} .
$$

It is immediately apparent that the sign of $\mathscr{C}_{0}$ and $\mathscr{C}_{1}$ is of particular importance in determining the type of bifurcation [13, 29]. Indeed a direct analysis for the existence of homogeneous solutions of this amplitude equation shows that the bifurcation will be subcritical for values of $\mathscr{C}_{1}>0$ and supercritical for values of $\mathscr{C}_{1}<0$. We plot the dependence of $\mathscr{C}_{0}$ and $\mathscr{C}_{1}$ on $\beta$ in Figure 3 and track the changes in sign that signify qualitative changes in physical behavior.

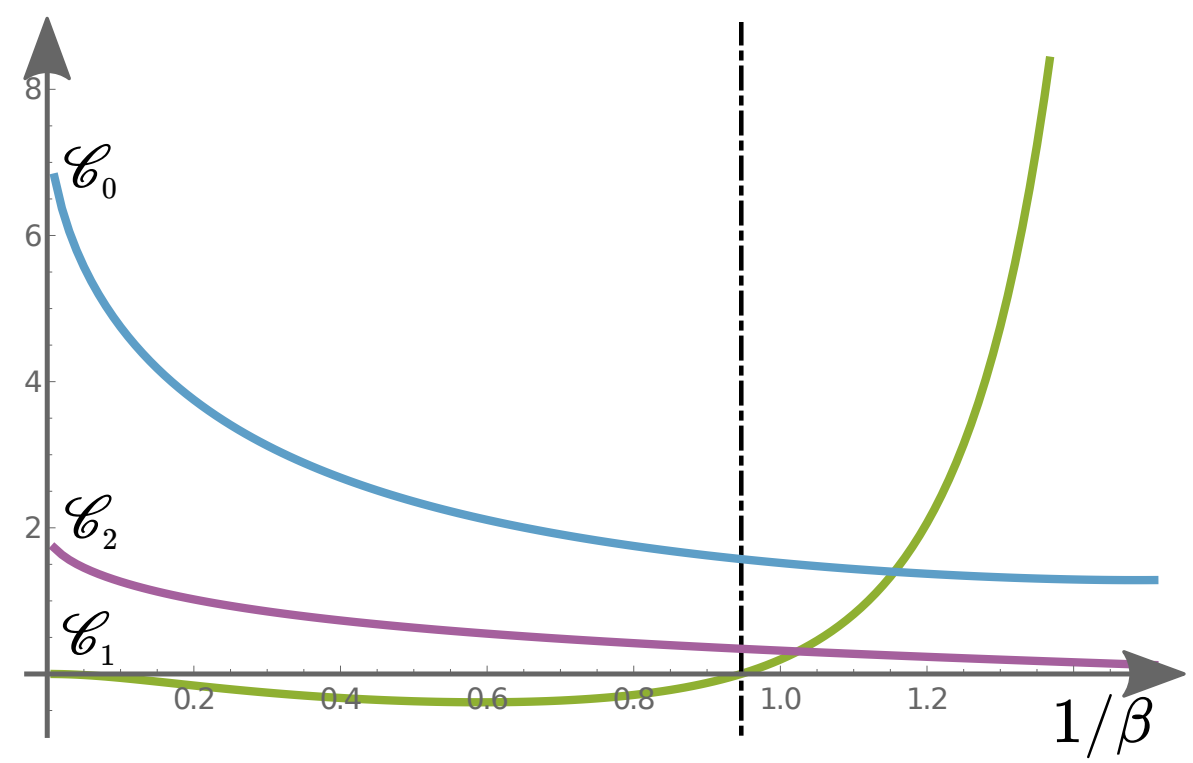

Figure 3: $\mathscr{C}_{0}, \mathscr{C}_{1}$ and $\mathscr{C}_{2}$ as functions of $\beta^{-1}$ in the growth case

Recalling that we must have $\beta>\beta_{\min } \approx 1.9^{-1}$ for the instability to take place, the bifurcation is subcritical for

$$
\beta_{\min }<\beta<0.95^{-1}
$$

and supercritical for

$$
\beta>0.95^{-1}
$$

We can now focus on the particular parameter value $\beta_{\mathrm{ss}} \approx 0.95^{-1}$ where the bifurcation goes from supercritical to subcritical. We return to the critical solutions of the dispersion relation illustrated in 
Figure 2 and identify the critical growth factor $\gamma_{\mathrm{ss}} \approx 1.576$ associated with $\beta_{\mathrm{ss}}$ (shown in Figure 4).

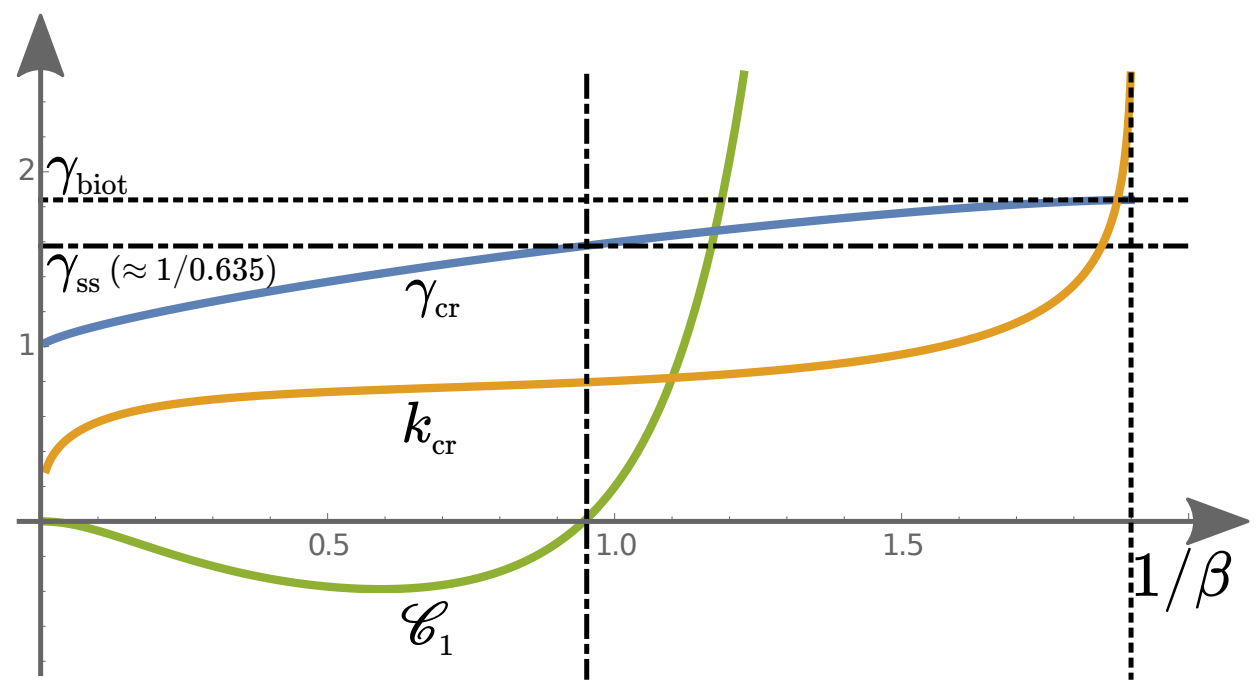

Figure 4: Growth factor $\gamma_{\mathrm{ss}}$ at the super-to-sub transition

\subsubsection{On creasing}

It has long been known that homogeneous soft elastic solids under compressive stress will undergo a creasing instability - the development of localized, self-contacting cusps on the free surface - rather than a wrinkling instability, thus avoiding the quandary of wrinkling length scale posed by Biot [8]. It has been well documented in the experimental and numerical literature that for bilayers with a sufficiently soft upper layer, we also see a creasing instability (see Wang and Zhao [53]).

At the point of the super-to-sub transition, the strain in the upper layer of the homogeneous trivial solution is given by $1-\gamma_{\mathrm{ss}}^{-1} \approx 0.365$. This is noteworthy by virtue of the fact that it corresponds almost exactly to the critical strain associated with some experimental studies on creasing in soft polymers [52, 51] (a summary of significant results in the literature can be found in Table 1). While our analysis does not yet constructively say anything about the formation of creases, the coincidence of the computed value of strain at the point at which wrinkling becomes unstable and the observed value for strain for which we expect a homogeneous medium to crease suggests that these phenomena might be related, or at least that these two instabilities may interact in the nonlinear regime.

\subsubsection{Verification of the amplitude relation}

Clearly, $a \equiv 0$ is always a solution of (3.21). For values of $\gamma_{1}$ close to 0 in the supercritical case, we expect $a$ to remain small and so we can then neglect the cubic term in our examination of the amplitude near the bifurcation point. Hence, in the static case we are interested in solutions of the linear ODE

$$
\mathscr{C}_{2} \partial_{\zeta \zeta} a+\mathscr{C}_{0} \gamma_{1} a=0
$$

which has general solution

$$
a(\zeta)=c_{1} \sin \left(\sqrt{\mathscr{C}_{0} \mathscr{C}_{2}^{-1} \gamma_{1}} \zeta\right)+c_{2} \cos \left(\sqrt{\mathscr{C}_{0} \mathscr{C}_{2}^{-1} \gamma_{1}} \zeta\right)
$$




\begin{tabular}{l|c|l} 
Reference & Creasing strain & Method \\
\hline$[22]$ & 0.364 & theory: asymptotic matching \\
{$[18]$} & 0.362 & theory: asymptotic matching \\
{$[39]$} & $0.35-0.36$ & simulation: finite element \\
{$[23]$} & 0.35 & simulation: finite element \\
{$[34]$} & 0.35 & simulation: finite element \\
{$[35]$} & 0.354 & simulation: finite element \\
{$[55]$} & 0.35 & simulation: finite element \\
{$[37]$} & 0.35 & simulation: finite element \\
{$[51]$} & $0.33-0.38$ & experiment: PDMS \\
{$[53$} & 0.36 & experiment: elastomer \\
{$[39$} & 0.37 & experiment: PDMS \\
{$[52]$} & 0.33 & experiment: hydrogel
\end{tabular}

Table 1: Previous results on strain required for creasing

On examination of the linear order of the periodic decomposition of the stream function found in (3.3), we see that the periodic behavior is modulated by the changing amplitude. More precisely, through use of Euler's formula, we see that we can rewrite $\Psi^{(1)}$ as a sum of terms of the form

$$
c e^{\left( \pm k_{\mathrm{cr}} \pm \sqrt{\mathscr{C}_{0} \mathscr{C}_{2}^{-1} \gamma_{1}} \epsilon\right) i x} h^{(1,1)}(Y) .
$$

Thus, the change in wavenumber $\delta:=\epsilon^{-1}\left(k-k_{\mathrm{cr}}\right)$ is related to the change in growth factor by the relation

$$
\delta^{2}=\frac{\mathscr{C}_{0}}{\mathscr{C}_{2}} \gamma_{1}
$$

We can compute the relationship between $\delta$ and $\gamma_{1}$ in an alternate manner by returning to the dispersion ratio. If we perturb (2.6) by changing $\gamma$ as prescribed in 3.2 and $k$ as $k=k_{\mathrm{cr}}+\epsilon \delta$, at second order in $\epsilon$ we obtain a closed form expression of the same form as (3.28). While it is computationally intractable to verify their equality analytically due to the form of $\mathscr{C}_{0}$ and $\mathscr{C}_{1}$, we can show their excellent agreement numerically.

\subsection{Compression case}

We can perform the same computations in the case of pure lateral compression. We perturb the critical compression ratio

$$
\lambda=\lambda_{\mathrm{cr}}\left(1+\epsilon^{2} \lambda_{1}\right)
$$

and take the same periodic decompositions 3.3 . The Euler-Lagrange equations for the first mode at second order now read

$$
\begin{aligned}
& \lambda_{\mathrm{cr}}^{2} \frac{\mathrm{d}^{4} h_{\mathrm{f}}^{(2,1)}}{\mathrm{d} Y^{4}}-k^{2}\left(1+\lambda_{\mathrm{cr}}^{4}\right) \frac{\mathrm{d}^{2} h_{\mathrm{f}}^{(2,1)}}{\mathrm{d} Y^{2}}+\lambda_{\mathrm{cr}}^{2} k^{4} h_{\mathrm{f}}^{(2,1)}=-2 i k\left(1+\lambda_{\mathrm{cr}}^{4}\right) \frac{\mathrm{d}^{2} h_{\mathrm{f}}^{(1,1)}}{\mathrm{d} Y^{2}}+4 i k^{3} \lambda_{\mathrm{cr}}^{2} h_{\mathrm{f}}^{(1,1)}, \\
& \lambda_{\mathrm{cr}}^{2} \frac{\mathrm{d}^{4} h_{\mathrm{s}}^{(2,1)}}{\mathrm{d} Y^{4}}-k^{2}\left(1+\lambda_{\mathrm{cr}}^{4}\right) \frac{\mathrm{d}^{2} h_{\mathrm{s}}^{(2,1)}}{\mathrm{d} Y^{2}}+\lambda_{\mathrm{cr}}^{2} k^{4} h_{\mathrm{s}}^{(2,1)}=-2 i k\left(1+\lambda_{\mathrm{cr}}^{4}\right) \frac{\mathrm{d}^{2} h_{\mathrm{s}}^{(1,1)}}{\mathrm{d} Y^{2}}+4 i k^{3} \lambda_{\mathrm{cr}}^{2} h_{\mathrm{s}}^{(1,1)}
\end{aligned}
$$

and the family of linear differential operators corresponding to 3.10 is given by

$$
\mathcal{L}_{\mathrm{f}}^{(n)}[h]=\mathcal{L}_{\mathrm{s}}^{(n)}[h]:=\lambda_{\mathrm{cr}}^{2} \frac{\mathrm{d}^{4} h}{\mathrm{~d} Y^{4}}-(n k)^{2}\left(1+\lambda_{\mathrm{cr}}^{4}\right) \frac{\mathrm{d}^{2} h}{\mathrm{~d} Y^{2}}+\lambda_{\mathrm{cr}}^{2}(n k)^{4} h .
$$


The general solutions of the Euler-Lagrange equations at second order are given by

$$
\begin{aligned}
& h_{\mathrm{f}}^{(2,1)}(Y)=c_{1}^{(2,1)} e^{-k \lambda_{\mathrm{cr}}^{-1} Y}+c_{2}^{(2,1)} e^{k \lambda_{\mathrm{cr}}^{-1} Y}+c_{3}^{(2,1)} e^{-k \lambda_{\mathrm{cr}} Y}+c_{4}^{(2,1)} e^{k \lambda_{\mathrm{cr}} Y}+\mathscr{G}_{\mathrm{f}}^{(2,1)}, \\
& h_{\mathrm{s}}^{(2,1)}(Y)=c_{5}^{(2,1)} e^{k \lambda_{\mathrm{cr}}^{-1} Y}+c_{6}^{(2,1)} e^{k \lambda_{\mathrm{cr}} Y}+\mathscr{G}_{\mathrm{s}}^{(2,1)},
\end{aligned}
$$

and

$$
\begin{aligned}
& h_{\mathrm{f}}^{(2,2)}(Y)=c_{1}^{(2,2)} e^{-2 k \lambda_{\mathrm{cr}}^{-1} Y}+c_{2}^{(2,2)} e^{2 k \lambda_{\mathrm{cr}}^{-1} Y}+c_{3}^{(2,2)} e^{-2 k \lambda_{\mathrm{cr}} Y}+c_{4}^{(2,2)} e^{2 k \lambda_{\mathrm{cr}} Y}+\mathscr{G}_{\mathrm{f}}^{(2,2)}, \\
& h_{\mathrm{s}}^{(2,2)}(Y)=c_{5}^{(2,2)} e^{2 k \lambda_{\mathrm{cr}}^{-1} Y}+c_{6}^{(2,2)} e^{2 k \lambda_{\mathrm{cr}} Y}+\mathscr{G}_{\mathrm{s}}^{(2,2)},
\end{aligned}
$$

where each $\mathscr{G}$ is a scalar, nonlinear function of $k(\beta)$ and $\lambda_{\mathrm{cr}}(\beta)$. Repeating the previously detailed procedure for the third order equations yields an amplitude relation of the form

$$
\partial_{\tau \tau} a-\mathscr{C}_{2} \partial_{\zeta \zeta} a=\mathscr{C}_{0} \lambda_{1} a+\mathscr{C}_{1}|a|^{2} a .
$$

Once more, supercritical solutions correspond to negative values of $\mathscr{C}_{1}$ (note that we are concerned with negative values of $\lambda_{1}$ ). Plots of the coefficients $\mathscr{C}_{0}$ and $\mathscr{C}_{1}$ are given in Figure 5.

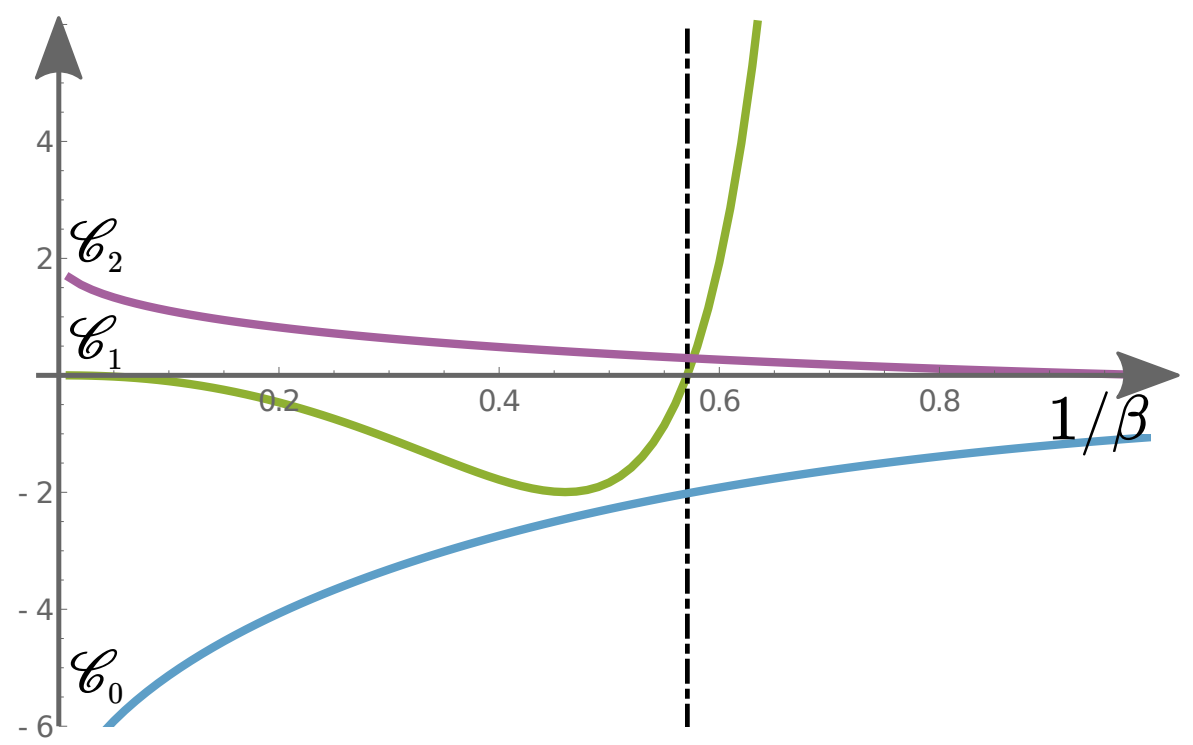

Figure 5: $\mathscr{C}_{0}, \mathscr{C}_{1}$ and $\mathscr{C}_{2}$ as functions of $\beta^{-1}$ in the compression case

We see that the transition from supercritical to subcritical behavior with decreasing $\beta$ occurs at $\beta_{\mathrm{SS}} \approx 0.571^{-1}\left(\beta_{\mathrm{SS}}^{-1} \approx 1.75\right)$, which corresponds to a critical compression ratio of $\lambda_{\mathrm{SS}} \approx 0.6747$. Our computed value of $\beta_{\mathrm{ss}}$ is close to, but differs slightly from the previously reported values of 1.74 [13] and 1.73 [38]. The difference may be due to a difference in the ansatz that we use (the base solutions are not strictly equivalent since we use a mixed-coordinates system). It should also be noted that the critical compression ratio agrees less well with the critical strain reported in creasing experiments. However, this is still consistent with these experimental observations - we show that when the wrinkling instability is stable, the critical strain required for wrinkling is always lower than the experimentally reported strain required for creasing

We have now successfully characterized the behavior of the wrinkling amplitude in the immediate post-buckling regime, but to probe the behavior of solutions further past the bifurcation point, we 
turn to numerical tools.

\section{Numerical bifurcation analysis}

In addition to the analytical calculations presented above, we carried out a systematic program of numerical experiments to explore the post-buckling regime in the growth case. In contrast to the approach used above, these finite element method computations were carried out without the use of the stream function construction - while this made the analysis more tractable, the full nonlinearity it introduces into (2.5) makes it unattractive for discretization. Instead, we adopted a mixed displacement-pressure formulation and imposed the incompressibility constraint with a Lagrange multiplier.

Another difference between the approaches comes from the nature of the domain. In our analytical calculations, we made use of a semi-infinite domain as an idealized model of the physical situation. Discretizing such domains is subtle, so we choose to truncate it in the $Y$ direction at some point. Various numerical studies [15, 39] suggest that a substrate ten times thicker than the film is sufficiently deep to negate any possible interaction between the bottom of the substrate and any wrinkles on the surface of the film as we expect the displacements to decay exponentially in the negative $Y$ direction. Additionally, we must choose a boundary condition to apply to the bottom of the substrate, which we choose to be zero displacement (although zero traction might also be appropriate). On the sides of the domain, we allow the material to slide freely up the "walls" of the domain, but not to penetrate through them (the displacement in the $X$ direction must vanish). A further difficulty arises from the width of the domain: in our analysis, we were able to choose this a posteriori to fit an integer number of wrinkles into it - a luxury we are not afforded in the numerical setting. We can inform our choice of domain size using the results of our perturbation analysis, but this will preclude us from independently verifying it.

Based on the above considerations, we restate our minimization problem:

$$
\underset{\substack{\mathbf{u} \in \boldsymbol{\Upsilon} \\ p \in L^{2}(\mathcal{B} ; \mathbb{R})}}{\operatorname{minimise}} I(\mathbf{u}, p):=\int_{\mathcal{B}}\left(\frac{\mu}{2} \operatorname{det} \mathbf{G}\right)\left(\operatorname{tr} \mathbf{A} \mathbf{A}^{T}-\operatorname{tr} \mathbf{I}\right)-p(\operatorname{det} \mathbf{A}-1) \mathrm{d} \mathbf{X} .
$$

where $\mathcal{B}=(-L, L) \times(-10,1)$ for some $L>0, \mathbf{G}=g \mathbf{I}, \mathbf{A}=\mathbf{I}+\frac{\partial \mathbf{u}}{\partial \mathbf{X}}$ and the set of admissible displacements is given by

$$
\mathbf{\Upsilon}=\left\{\mathbf{u} \in H^{1}\left(\mathcal{B} ; \mathbb{R}^{2}\right): \mathbf{u}(\cdot,-10)=(0,0), u( \pm L, \cdot)=0\right\},
$$

where $\mathbf{u}(\mathbf{X})=(u(X, Y), v(X, Y))$ and evaluation on the boundary is understood in the sense of traces. As usual, we compute solutions of (4.1) by solving the Euler-Lagrange equation

$$
\delta I=0
$$

varying in $\mathbf{u}$ and $p$ simultaneously.

After unreported numerical experiments, we settled on using the Scott-Vogelius $\left(C G_{k}\right)^{2} \times D G_{k-1}$ mixed finite element on an unstructured triangular mesh with a single iteration of barycentric mesh refinement applied to it, yielding a total of 141,730 degrees of freedom for each PDE solve for $k=2$. The mesh was graded in such a way that the vast majority of resolution was concentrated in the film; the predicted exponential decay in the displacement in the substrate allowed for a very coarse mesh towards the bottom of the computational domain. The choice of finite element discretization comes from both considerations of stability and effective imposition of the nonlinear incompressibility 
condition. Braess and Ming [10] demonstrated that any pair of spaces that is stable for the Stokes problem will also be stable for incompressible hyperelasticity. The Scott-Vogelius element pair has been shown by Arnold and Qin [3] to be stable for the 2D Stokes problem on barycentrically refined meshes for $k \geq 2$ and enjoys a number of advantageous properties (see Olshanskii and Rebholz [48]) such as exact imposition of the divergence-free constraint found in the incompressible formulation of the problem. While achieving exact discrete enforcement of the nonlinear constraint in this formulation is not possible, we noted a two order of magnitude reduction in the constraint residual

$$
\|\operatorname{det} \mathbf{A}-1\|_{L^{2}(\mathcal{B} ; \mathbb{R})},
$$

compared to the more common Taylor-Hood element. For a detailed exposition of the substantial effects that poor enforcement of the incompressibility constraint can have on the computation of bifurcation points for problems in elasticity, we refer to Auricchio et al. [4].

\subsection{Computation of bifurcation diagrams}

We wish to understand the evolution of the solution set of the PDE as its parameters are varied. The main tool we apply is deflated continuation, an algorithm that advantageously combines two existing techniques in nonlinear PDE analysis: deflation and continuation.

Deflation can be summarized as the positive answer to the following question:

Given a solution $z^{*}$ to a nonlinear PDE problem $\mathcal{F}$, can we (under reasonable conditions) define a new problem $\mathcal{G}$ that satisfies the conditions below?

1. applying Newton's method to $\mathcal{G}$ will not yield $z^{*}$;

2. for $z \neq z^{*}, z$ solves $\mathcal{F} \Leftrightarrow z$ solves $\mathcal{G}$.

This is achieved by systematically introducing singularities to the problem that will prevent the rediscovery of $z^{*}$ by Newton's method. This technique was developed by Farrell, Birkisson and Funke [25] as an adaptation of the approach developed by Brown and Gearheart [11] for the solution of nonlinear algebraic equations.

Continuation methods are a classical tool in bifurcation analysis. Given a nonlinear equation

$$
\mathcal{F}(z, \gamma)=0
$$

with parameter $\gamma \in \mathbb{R}$, suppose we have found a solution $z_{0}^{*}$ for a particular $\gamma_{0}$ and wish to now solve the equation for a slightly perturbed parameter value, say $\gamma_{1}=\gamma_{0}+\Delta \gamma$. There are several approaches to this problem, the most obvious of which is zero-order continuation. For this, we simply use $z_{0}^{*}$ as the initial guess for the solution at $\gamma=\gamma_{1}$.

The two procedures are combined as follows. Given a bifurcation problem 4.5), we specify a range of parameter values $\left[\gamma_{\min }, \gamma_{\max }\right]$ of interest and a continuation step size $\Delta \gamma$. Then, starting from $\gamma=\gamma_{\min }$, we first perform zero-order continuation followed by deflation on each of the known solutions for the current value of $\gamma$. We then search for new solutions using Newton's method, with one initial guess for each of the previous solutions. If we find another solution, we can deflate it and repeat our search from the same initial guess until Newton's method fails to converge. Finally, we increment $\gamma$ by $\Delta \gamma$ and repeat the whole process until we reach $\gamma_{\max }$. The main advantage of this approach is that it allows for the detection of disconnected branches, so long as they are close to known ones. 


\subsection{Numerical results}

We discretized and numerically solved the Euler-Lagrange equation (4.3) using Firedrake [49] and PETSc [5]. We considered 19 values of the inverse stiffness ratio $\beta^{-1}$ between 0.05 and 0.95 . For each fixed value of $\beta$, we applied deflated continuation in $\gamma$ for an appropriate subset of $[1,1.6]$. We used a non-uniform spacing of values with the density of continuation points increasing with proximity to an asymptotic approximation of the critical growth value $\gamma_{\mathrm{cr}}$. Each solve of the nonlinear PDE was performed using Newton's method, with the linear system arising at each Newton iteration solved using the sparse LU factorization algorithm of MUMPS [2].

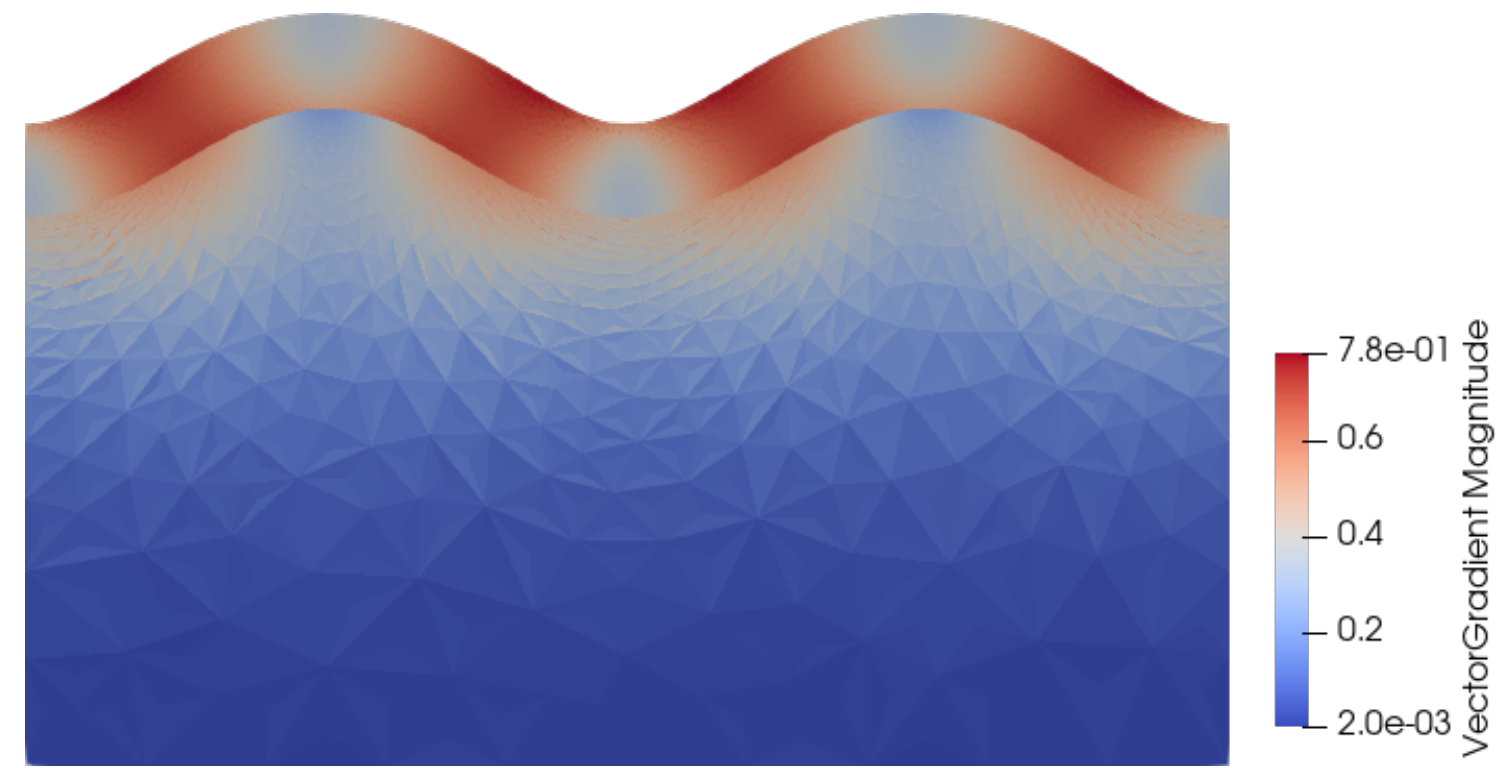

Figure 6: Example numerical wrinkling solution for $\beta=4, \gamma=1.303$ (colored by strain-we can see the mesh because the strain approximation is discontinuous)

We were able to discover the primary wrinkling bifurcation and continue solutions past the critical growth parameter for the full range of stiffness ratios considered. An example of a post-bifurcation wrinkling solution for an intermediate value of $\beta$ is shown in Figure 6. For each stiffness ratio, we can then compute the evolution of the observed wrinkling amplitude of the numerical solutions as $\gamma$ is increased past $\gamma_{\text {cr }}$ by computing

$$
a_{\mathrm{obs}}=\|v\|_{L^{\infty}(\{Y=1\} ; \mathbb{R})}-\left(\gamma^{2}-1\right) .
$$

This was then compared (see Appendix B) to the corresponding solutions of the static amplitude equation (3.22) (an example is shown in Figure 7) and found to be in excellent agreement with no fitting parameters in most cases. However, for some stiffness ratios we found that the bifurcation did not occur until slightly after the predicted critical value of $\gamma$ (although the shape of the bifurcating branch remained correct). To investigate this phenomenon further, we turned to direct methods for determining where the first instance of a non-trivial solution branch occurs in the numerics.

\subsection{Direct computation of the primary bifurcation point}

While the computations described in the previous section allow us to examine the post-buckling behavior of the system past the bifurcation point, it is somewhat difficult to see precisely where the pitchfork begins. However, we can identify the bifurcation point of the discretized system by solving 


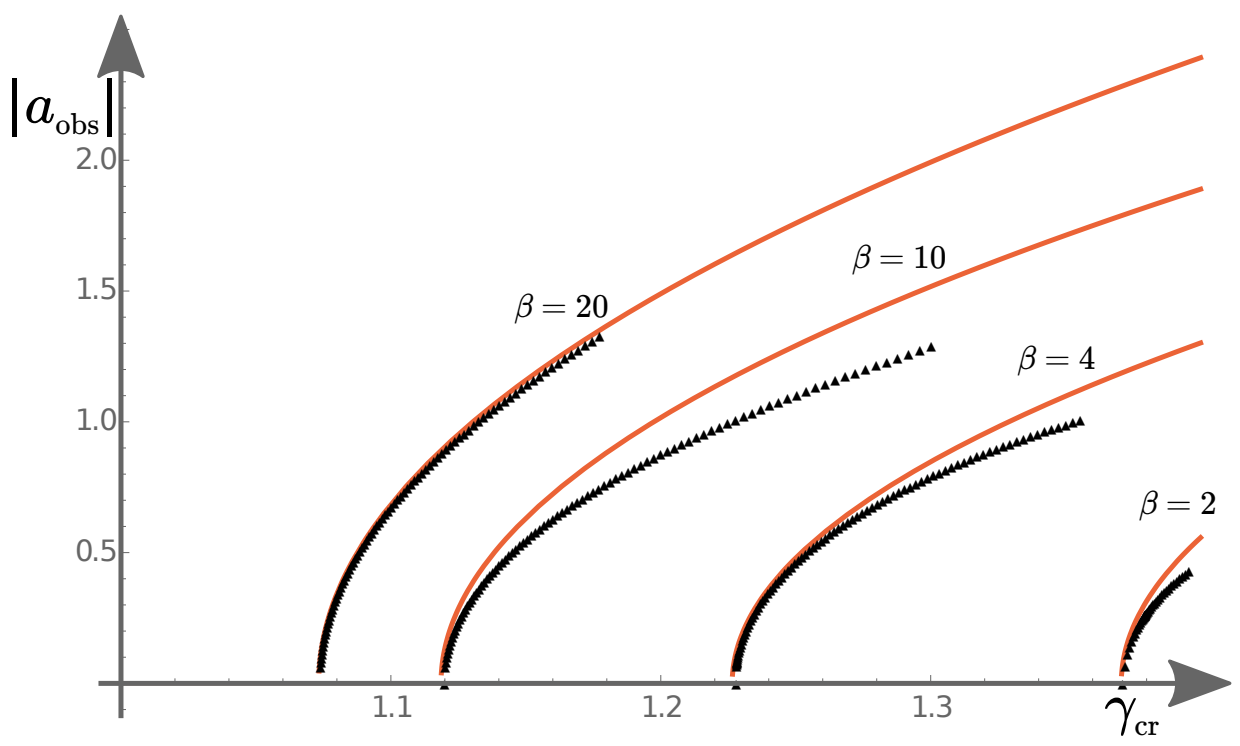

Figure 7: Observed amplitude of numerical solutions (black) and amplitude relation (3.22) (orange) for $\beta \in\{20,10,4,2\}$. The amplitude relation (which is valid for a small perturbation of $\gamma_{\mathrm{cr}}$ ) shows good agreement with the numerics well into the post-buckling regime.

an extended set of equations known as the Moore-Spence system (introduced by Moore and Spence [47]).

The essential idea is to treat the bifurcation parameter $\gamma$ in 4.5 as a variable we wish to solve for and augment the equation with the requirement that the derivative of the residual in the $z$ direction is not invertible (and hence has a normalized eigenvector $w$ with eigenvalue 0 ). Mathematically the combined system can be written as: find $z, \gamma, w \in Z \times \mathbb{R} \times Z$ such that

$$
\begin{aligned}
\mathcal{F}(z, \gamma) & =0 \\
\mathcal{F}_{z}(z, \gamma) w & =0 \\
\|w\| & =1
\end{aligned}
$$

In order to solve a discretization of this system using Newton's method, an initial guess must be carefully constructed. First, we solve (4.7a) for $z$ using the following asymptotic approximation for $\gamma_{\mathrm{cr}}$ for our chosen value of $\beta$ (see [1] for its derivation):

$$
\gamma_{\mathrm{cr}}^{2} \approx 1+\frac{3^{2 / 3}}{2} \beta^{-2 / 3}+\frac{2 \cdot 3^{1 / 3}}{5} \beta^{-4 / 3}+\frac{201}{2800} \beta^{-2}
$$

We then use this computed $z$ to solve the generalized eigenvalue problem

$$
\mathcal{F}_{z}(z, \gamma) w=r w
$$

for the eigenmode $w$ associated with the minimal eigenvalue $r$ using the SLEPc [33] implementation of the Krylov-Schur algorithm [50]. We can then combine our approximate $z, \gamma_{\mathrm{cr}}$ and $w$ to form an initial guess for Newton's method applied to the complete Moore-Spence system (4.7). 


\subsubsection{Effect of computational domain aspect ratio}

Due to the finite width of our computational domain and our enforcement of sliding boundary conditions on the vertical walls, we are constrained to only ever realize an integer number of wrinkling periods in the numerical solutions we find. This both makes it difficult to verify precisely the predictions regarding wavenumber from the linear analysis and has the effect of delaying the bifurcation slightly past the predicted critical growth threshold. To examine this effect more closely, we can introduce an aspect ratio parameter $\alpha$ in order to transform the computational domain from width $2 L$ to $2 \alpha L$ while keeping the height of both layers the same. We apply the simple coordinate transform

$$
\begin{aligned}
X & =\alpha^{-1} \tilde{X} & Y & =\tilde{Y} \\
u(X, Y) & =\alpha^{-1} \tilde{u}(\tilde{X}, \tilde{Y}) & v(X, Y) & =\tilde{v}(\tilde{X}, \tilde{Y}),
\end{aligned}
$$

which gives us the deformation gradient relation

$$
\begin{aligned}
\frac{\partial \tilde{u}}{\partial \tilde{X}}=\frac{\partial u}{\partial X} & \frac{\partial \tilde{u}}{\partial \tilde{Y}}=\alpha \frac{\partial u}{\partial Y} \\
\frac{\partial \tilde{v}}{\partial \tilde{X}}=\alpha^{-1} \frac{\partial v}{\partial X} & \frac{\partial \tilde{v}}{\partial \tilde{Y}}=\frac{\partial v}{\partial Y} .
\end{aligned}
$$

Using these relations, we can replace the appropriate terms in (4.1) to formulate a minimization problem for the rescaled system. This approach carries the significant benefit of allowing us to perform solves for domains of different aspect ratios without the tedious process of re-meshing.

Equally, we can also use this rescaling with the discretized Moore-Spence system to efficiently examine the effect of domain width on the observed bifurcation point in the numerical system. Of particular interest is the observed wavenumber $k_{\text {obs }}$ of a numerical wrinkling solution given by

$$
k_{\mathrm{obs}}:=\frac{\left(n_{\mathrm{obs}}+1\right) \pi}{2 L \alpha},
$$

where $n_{\text {obs }}$ is the observed number of minima and maxima of the vertical displacement of the top surface of the domain (excluding the endpoints). Given a fixed stiffness ratio $\beta$ and corresponding critical wavenumber $k_{\mathrm{cr}}$, we can then compute the value of the aspect ratio $\alpha_{n}$ that would allow $(n+1) / 2$ periods (or equivalently $n$ interior extrema) of a sinusoidal profile with wavenumber $k_{\mathrm{cr}}$ to fit into the interval $[-\alpha L, \alpha L]$ :

$$
\alpha_{n}:=\frac{(n+1) \pi}{2 L k_{\mathrm{cr}}} .
$$

By solving the discretised Moore-Spence system for a range of values of $\alpha$, we see that those which minimize the gap between the expected and realized value of $\gamma_{\text {cr }}$ occur close to some $\alpha_{n}$ for an even value of $n$ (see Figure 8 for an example). This suggests that the delay in bifurcation is predominantly caused by the domain-width constraint. This effect is accentuated at lower values of $\beta$, as illustrated in Figure 9. As hinted at in Figure 8, even with the optimal aspect ratio, there remains a small but significant difference between the expected and observed value of $\gamma_{\mathrm{cr}}$ for some values of $\beta$. Some of this can be explained by discretization error and the domain truncation that we carry out, but we have not yet performed a comprehensive analysis on whether we can explicitly account for all of the difference as a result of these factors. Nonetheless, our numerical experiments have provided a robust corroboration of our weakly-nonlinear analysis and given us further insight into the postbuckling behavior of the system past the bifurcation point. In particular, the tools developed are easily adaptable to further numerical experiments such as different domain geometries, boundary conditions, or secondary bifurcation such as ridge formation or period doubling [46, 12, 27] that were 


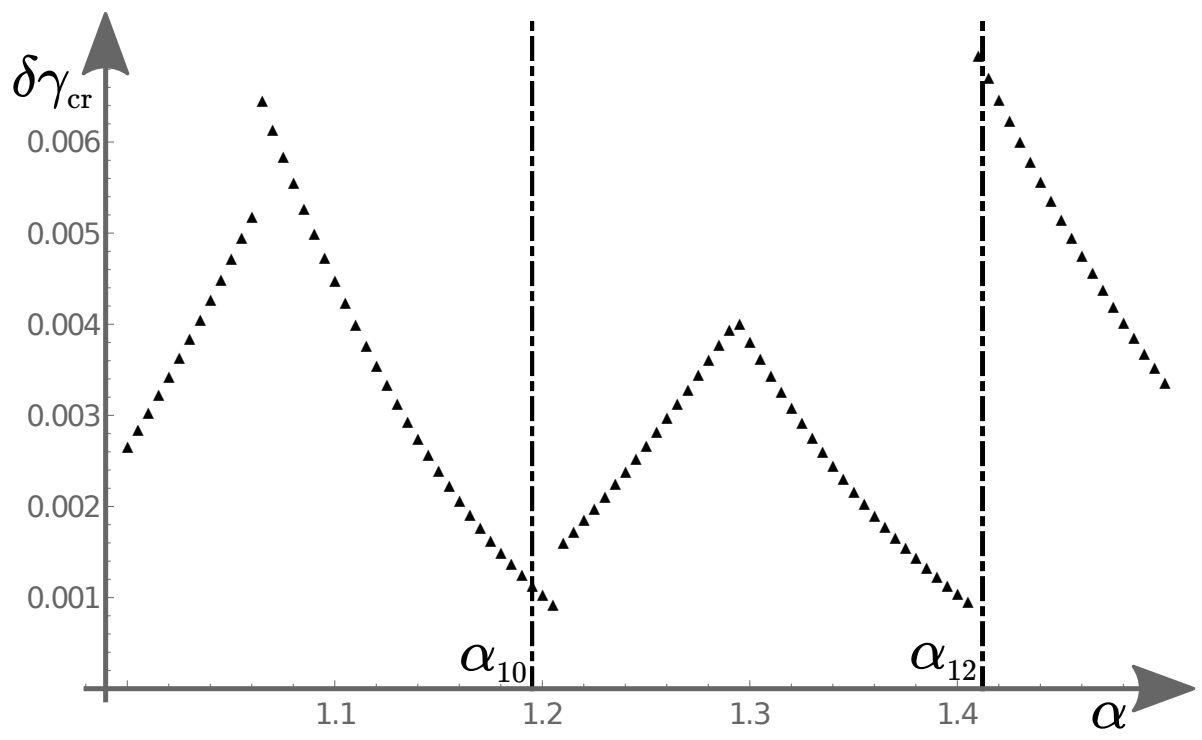

Figure 8: Error in computed bifurcation point as a function of aspect ratio $\alpha$ for $\beta=2.5$

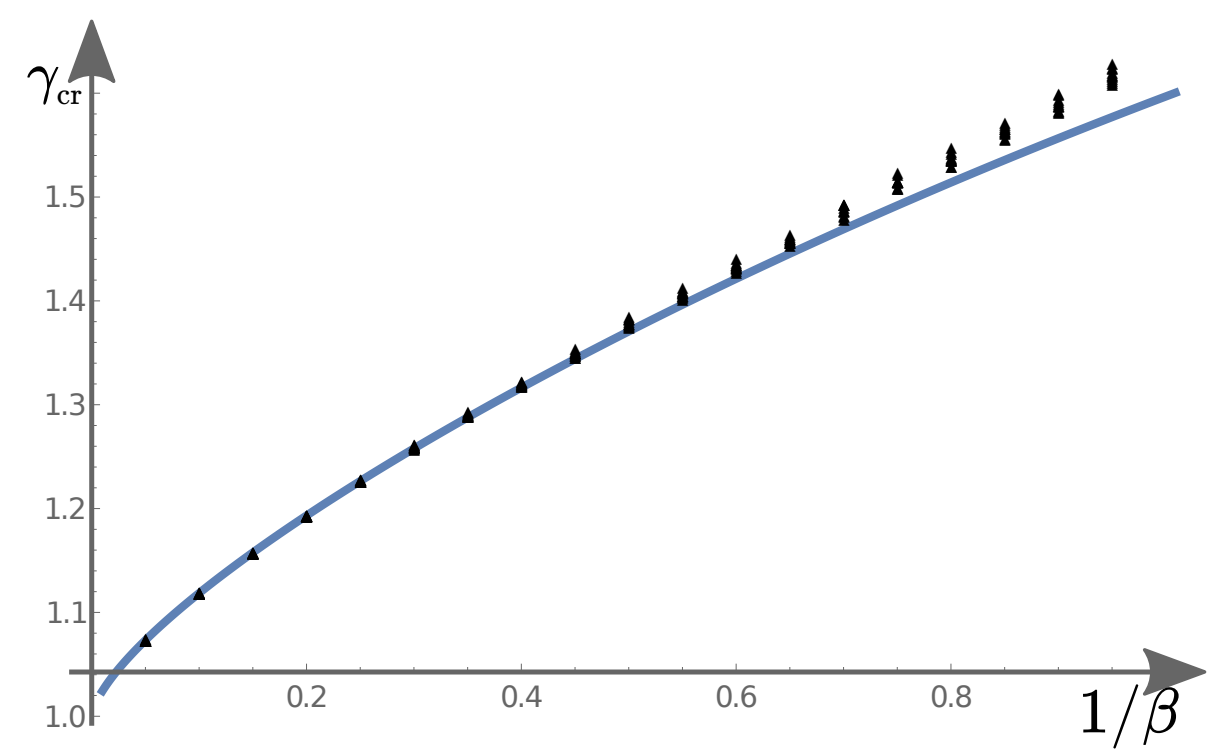

Figure 9: Computed critical growth factor as a function of $\beta^{-1}$. For each value of $\beta$ considered, we performed the computations for 11 uniformly spaced aspect ratios $\alpha \in[1,1.5]$ (all shown)

observed away from the bifurcation point.

\section{Solutions of the amplitude relation}

We can now search for solutions of the nonlinear PDE (3.21), following the method set out by Lega and Goriely [44]. First, let us consider traveling wave solutions for which

$$
a(\zeta, \tau)=\tilde{a}(\zeta-c \tau) e^{i \omega \tau}
$$


for some wave speed $c$ and phase shift $\omega$. Writing $\xi=\zeta-c \tau$, we then obtain

$$
\left(\mathscr{C}_{2}-c^{2}\right) \tilde{a}^{\prime \prime}+2 i c \omega \tilde{a}^{\prime}+\left(\mathscr{C}_{0} \gamma_{1}+\omega^{2}\right) \tilde{a}+\mathscr{C}_{1}|\tilde{a}|^{2} \tilde{a}=0 .
$$

This can be further transformed into a pair of real-valued equations by writing $\tilde{a}$ in polar form, i.e. $\tilde{a}(\xi)=r(\xi) e^{i \theta(\xi)}$, and taking real and imaginary parts of the resulting equation. Here it is useful to write the derivative of $\theta$ as $\Theta$

$$
\begin{aligned}
\left(\mathscr{C}_{2}-c^{2}\right) r^{\prime \prime}+\left(-2 c \omega \Theta\left(\mathscr{C}_{2}-c^{2}\right) \Theta^{2}+\mathscr{C}_{0} \gamma_{1}+\omega^{2}\right) r+\mathscr{C}_{1} r^{3} & =0, \\
-2\left(\left(\mathscr{C}_{2}-c^{2}\right) \Theta-c \omega\right) r^{\prime}+\left(\mathscr{C}_{2}-c^{2}\right) \Theta^{\prime} r & =0 .
\end{aligned}
$$

We can then find two first integrals of $(5.3)$ :

$$
\begin{aligned}
& \Phi_{1}=c\left(\mathscr{C}_{2}-c^{2}\right) \omega\left(r^{\prime}\right)^{2}+\left(\mathscr{C}_{2}-c^{2}\right) r^{2} \Theta\left(c \omega \Theta-\mathscr{C}_{0} \gamma_{1}-\omega^{2}\right)+\frac{1}{2} c \mathscr{C}_{1} \omega r^{4}, \\
& \Phi_{2}=-r^{2}\left(\left(\mathscr{C}_{2}-c^{2}\right) \Theta+c \omega\right) .
\end{aligned}
$$

The latter equation can be rearranged to provide a closed form expression for $\Theta$ in terms of $r$ :

$$
\Theta=-\frac{\Phi_{2}+c \omega r^{2}}{\left(\mathscr{C}_{2}-c^{2}\right) r^{2}}
$$

The two first integrals can be combined to write down a useful conserved quantity

$$
\Phi=\frac{\left(\mathscr{C}_{2}-c^{2}\right)\left(\Phi_{1}-\mathscr{C}_{0} \gamma_{1} \Phi_{2}\right)-\left(\mathscr{C}_{2}+c^{2}\right) \Phi_{2} \omega^{2}}{c\left(\mathscr{C}_{2}-c^{2}\right)^{2} \omega}
$$

In particular, we can interpret $\Phi$ as a Hamiltonian for the motion of a particle in a potential $V_{\text {eff }}$ defined as follows:

$$
\Phi-\left(r^{\prime}\right)^{2}=v_{-2} r^{-2}+v_{2} r^{2}+v_{4} r^{4}=: V_{\text {eff }},
$$

where the coefficients $v_{i}$ are given by:

$$
v_{-2}=\frac{\Phi_{2}^{2}}{\left(\mathscr{C}_{2}-c^{2}\right)^{2}}, \quad v_{2}=\frac{\mathscr{C}_{2}\left(\mathscr{C}_{0} \gamma_{1}+\omega^{2}\right)-c^{2} \mathscr{C}_{0} \gamma_{1}}{\left(\mathscr{C}_{2}-c^{2}\right)^{2}}, \quad v_{4}=\frac{\mathscr{C}_{1}}{2\left(\mathscr{C}_{2}-c^{2}\right)}
$$

Depending on the values of the parameters, the "potential" $V_{\text {eff }}$ can take a number of different shapes (illustrated in figs. 10 and 11), only some of which support periodic orbits. Of particular importance in determining this are signs of $v_{2}$ and $v_{4}$ as well as whether $v_{-2}$ vanishes. Let us restrict our attention to the static case $(c=\omega=0)$. We always have $\mathscr{C}_{0}>0$ and $\mathscr{C}_{2}>0$ and we see a sign change in $\mathscr{C}_{1}$ from negative for $\beta>\beta_{\mathrm{ss}}$ to positive for $\beta<\beta_{\mathrm{ss}}$. At the same point, we also see a change in the sign of $\gamma_{1}$ from positive to negative as the system changes from supercritical to subcritical with decreasing $\beta$. The coefficients of $V_{\text {eff }}$ are given by

$$
v_{-2}=\frac{\Phi_{2}^{2}}{\mathscr{C}_{2}^{2}}, \quad v_{2}=\frac{\mathscr{C}_{0} \gamma_{1}}{\mathscr{C}_{2}}, \quad v_{4}=\frac{\mathscr{C}_{1}}{2 \mathscr{C}_{2}}
$$

By considering the shape of $V_{\text {eff }}$, we see that if $\Phi_{2}$ (and hence $v_{-2}$ ) vanishes, we can find localized solutions if and only if $v_{2}$ and $v_{4}$ have opposite sign. By the observations above, we see that this is 
always true. If $\Phi_{2}$ is nonzero, we must have $v_{2}>0, v_{4}<0$ and $\sqrt{3 v_{2}^{3}}+9 v_{4}>0$ in order to see such

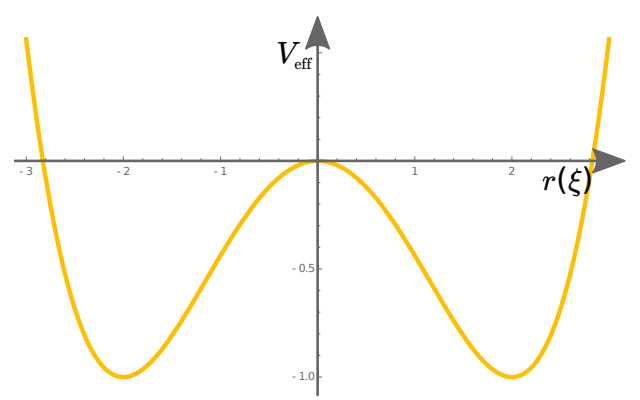

(a) $v_{2}<0, v_{4}>0$

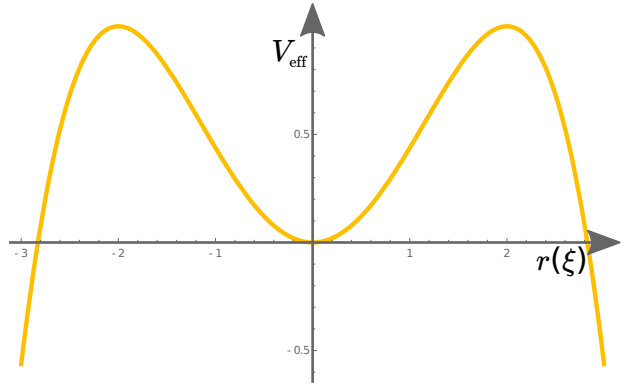

(b) $v_{2}>0, v_{4}<0$

Figure 10: Potentials for which $v_{-2}$ vanishes

solutions, which immediately limits us to the supercritical case. In our efforts to better understand

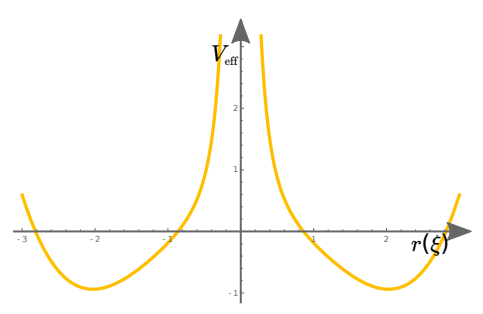

(a) $v_{2}<0, v_{4}>0$

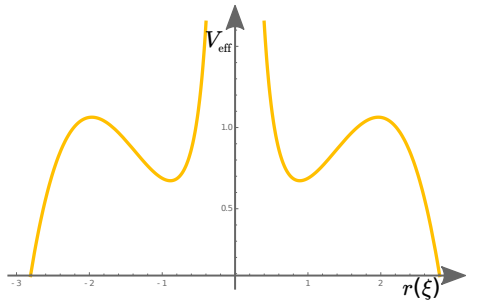

(b) $v_{2}>0, v_{4}<0, \sqrt{3 v_{2}^{3}}>-9 v_{4}$

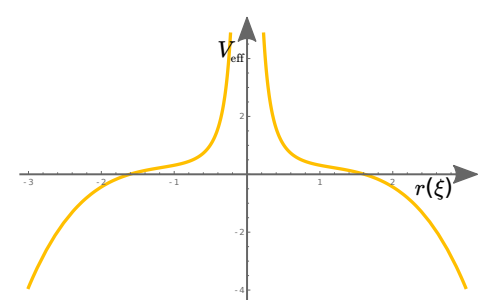

(c) $v_{2}>0, v_{4}<0, \sqrt{3 v_{2}^{3}}<-9 v_{4}$

Figure 11: Potentials for which $v_{-2}$ does not vanish

the subcritical case, let us focus on the case in which $v_{-2}=0, v_{2}<0$ and $v_{4}>0$. localized solutions in such potentials consist of homoclinic orbits linking $\tilde{r}=0$ to itself. The maximal value of $r$ achieved in such orbits can easily be seen to be

$$
r_{\max }=\sqrt{-\frac{v_{2}}{v_{4}}}=\sqrt{-\frac{2 \mathscr{C}_{0} \gamma_{1}}{\mathscr{C}_{1}}}
$$

\subsection{Explicit solutions}

In order to compute explicit solutions, it is convenient to make the substitution

$$
\tilde{r}(\xi)=-\left(\frac{v_{2}}{3}+v_{4} r(\xi)^{2}\right)
$$

The Hamiltonian (5.7) then becomes

$$
\left(\tilde{r}^{\prime}\right)^{2}=4 \tilde{r}^{3}-g_{2} \tilde{r}-g_{3},
$$

where the invariants $g_{i}$ are given by

$$
g_{2}=\frac{4}{3}\left(v_{2}^{2}+3 \Phi v_{4}\right), \quad g_{3}=-\left(\frac{8 v_{2}^{3}}{27}+\frac{4 \Phi v_{2} v_{4}}{3}+4 v_{4}^{2} v_{-2}\right)
$$


So long as $g_{2}^{3} \neq 27 g_{3}^{2}$, the general solution of 5.12 is given by

$$
\tilde{r}(\xi)=\wp\left(\xi+\xi_{0} \mid \omega_{1}, \omega_{3}\right),
$$

where $\wp$ is the Weierstrass elliptic function and $\xi_{0}$ is a constant of integration. To compute the half periods $\omega_{i}$, let us label the roots of the cubic on the right hand side of (5.12) as $e_{1}, e_{2}$ and $e_{3}$ so that it reads

$$
\left(\tilde{r}^{\prime}\right)^{2}=4\left(\tilde{r}-e_{1}\right)\left(\tilde{r}-e_{2}\right)\left(\tilde{r}-e_{3}\right) .
$$

If $g_{2}^{3}>27 g_{3}^{2}$, all three roots are real and distinct. In this case, let us order them so that $e_{1}>e_{2}>e_{3}$. We then have

$$
\begin{aligned}
\omega_{1} & =\int_{e_{1}}^{\infty}\left(4 \hat{r}^{3}-g_{2} \hat{r}-g_{3}\right)^{-1 / 2} \mathrm{~d} \hat{r}, \\
\omega_{3} & =i \int_{e_{3}}^{\infty}\left(4 \hat{r}^{3}-g_{2} \hat{r}-g_{3}\right)^{-1 / 2} \mathrm{~d} \hat{r}, \\
\omega_{2} & =-\left(\omega_{1}+\omega_{3}\right) .
\end{aligned}
$$

For a detailed review of the theory of elliptic functions in this context, refer to Whittaker and Watson [54]. Reversing the transformation (5.11), we can now write an expression for $r$ :

$$
r(\xi)=\sqrt{-\frac{v_{2}+3 \wp\left(\xi+\xi_{0} \mid \omega_{1}, \omega_{3}\right)}{3 v_{4}}} .
$$

At this stage, we make the important observation that in the static case, the first integrals (5.4) are actually scalar multiples of one another:

$$
\Phi_{1}=-\mathscr{C}_{0} \mathscr{C}_{2} \gamma_{1} r^{2} \Theta, \quad \Phi_{2}=-\mathscr{C}_{2} r^{2} \Theta .
$$

If $\Phi_{2}$ vanishes, $\Phi_{1}$ (and hence $\left.\Phi\right)$ must also vanish and either $r \equiv 0$ or $\theta$ is constant (i.e. it just has the effect of a uniform phase shift). The vanishing of both $\Phi$ and $\Phi_{2}$ means we now have $g_{2}^{3}=27 g_{3}^{2}$ and $\omega_{3}=\infty$. In this special case, the Weierstrass elliptic function and its imaginary half period $\omega_{1}$ can be expressed in the following elementary fashion:

$$
\wp\left(\xi \mid \omega_{1}, \infty\right)=\left(\frac{\pi}{2 \omega_{1}}\right)^{2}\left(\frac{1}{\sin ^{2}\left(\frac{\pi \xi}{2 \omega_{1}}\right)}-\frac{1}{3}\right), \quad \omega_{1}^{2}=\frac{g_{2} \pi^{2}}{18 g_{3}}=\frac{\mathscr{C}_{2} \pi^{2}}{4 \mathscr{C}_{0} \gamma_{1}} .
$$

We can now use (5.17) and (5.19) to write down an explicit form for a localized solution in the subcritical case $v_{-2}=0, v_{2}<0, v_{4}>0$. Taking $\xi_{0}$ to be an odd multiple of $\omega_{1}$ in (5.14) to ensure a real-valued solution, we then obtain

$$
r(\xi)=\sqrt{-\frac{2 \mathscr{C}_{0} \gamma_{1}}{\mathscr{C}_{1}}} \operatorname{sech}\left(\sqrt{-\frac{\mathscr{C}_{0} \gamma_{1}}{\mathscr{C}_{2}} \xi}\right) .
$$

Finally, we return to our perturbation expansion $(3.1)$ and periodic decomposition (3.3) to realize mathematically the profile of a variable amplitude solution to our system, an example of which is shown graphically in Figure 12.

In the subcritical case that supports localized solutions $\left(v_{-2}=0, v_{2}<0, v_{4}>0\right)$, we have $g_{2}^{3}>27 g_{3}^{2}$ for all positive values of $\Phi$. Closer inspection of the $\omega_{i}$ show that for values of $\Phi$ sufficiently close to 0 , the period of the homoclinic orbits described in the previous section can be made arbitrarily large. 


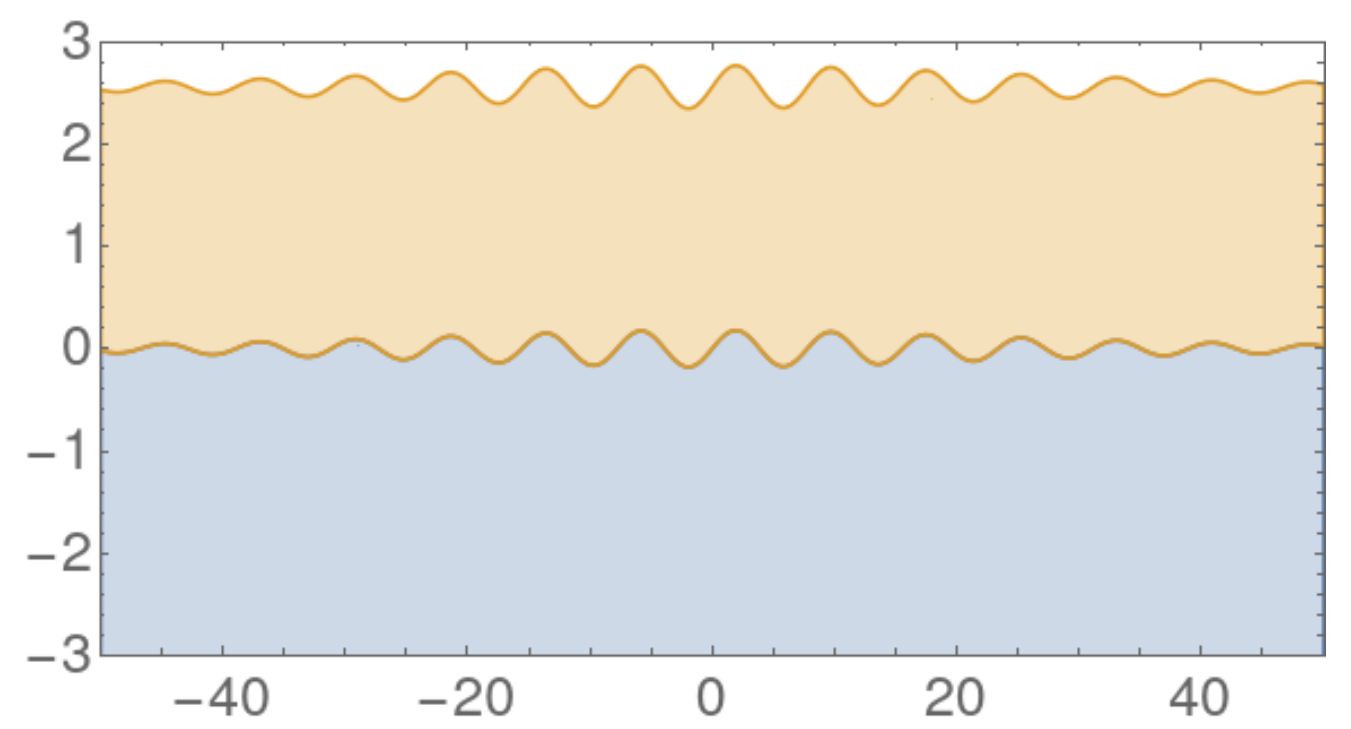

Figure 12: Profile of a subcritical variable amplitude solution for $\beta^{-1}=1.01, \gamma=\gamma_{\mathrm{cr}}-0.01^{2}$. Note that the aspect ratio is not $1: 1$ and has been chosen to demonstrate amplitude modulation on the long horizontal scale

In the limiting case, the solution would become entirely localized, corresponding to a deformation concentrated at a point. While it is attractive to consider that creasing phenomena could perhaps be understood in this fashion, significant further investigation and interpretation is required before any such conclusions can be made.

\section{Conclusion}

This paper gives a detailed exposition of the post-buckling behavior of an elastic film bonded to an elastic substrate and subjected to compressive stress induced by either growth of the film or lateral compression. First, a weakly-nonlinear analysis was carried out to derive an amplitude equation that describes how the magnitude of surface oscillations increases past the critical growth factor. Analysis of this equation also yielded a threshold value for the transition between a supercritical and subcritical bifurcation when the film becomes sufficiently soft relative to the substrate. These analyses were corroborated by a numerical bifurcation analysis using a carefully chosen finite element discretization, which obtained good agreement with the post-buckling amplitude without fitting parameters. A numerical scheme to directly compute the bifurcation point of the system was described and used to investigate the effect of domain aspect ratio on the observed value of the critical growth factor in the computations. Finally, we were able to characterize qualitative behaviors of non-constant solutions to the amplitude equations, give an explicit general solution in terms of an elliptic function and realize a variable-amplitude solution of our system in the subcritical regime that features completely localized deformation. One limitation of our work is the restriction of our analyses to the case of uniaxial compression in plane strain. The stream function approach does not easily generalise to the fully three-dimensional description required for the study of biaxial compression. In this case, recent progress has been made in works such as [16] by analysis of the original equations, but this forgoes the benefits of exact imposition of the incompressibility constraint that the stream function formulation provides. While aspects of our analysis appear similar to previous works, we believe that a detailed post-bifurcation analysis validated with a dedicated numerical scheme is necessary for the further development of the field. In particular, outstanding questions regarding the long-time behavior of the 
subcritical bifurcation branches and secondary bifurcations [12, 26] such as period-doubling and ridge formation remain open.

\section{Acknowledgements}

This work was supported by the Engineering and Physical Sciences Research Council grants EP/R020205/1 (Alain Goriely), EP/R029423/1 (Patrick Farrell) and EP/L015811/1 (Hamza Alawiye). We thank Prof. Simon Tavener and Dr. Jessica Williams for productive discussions regarding the effects of the aspect ratio of the computational domain on the results of our simulations.

\section{Appendix A. Boundary conditions}

The boundary and decay conditions for the Euler-Lagrange equations 2.5 are given by:

$$
\begin{aligned}
& \frac{\partial \Psi_{\mathrm{f}}}{\partial x}=\frac{\partial \Psi_{\mathrm{s}}}{\partial x} \\
& \frac{\partial \Psi_{\mathrm{f}}}{\partial Y}=\gamma^{2} \frac{\partial \Psi_{\mathrm{s}}}{\partial Y} \\
& \frac{\partial}{\partial Y}\left(\frac{\partial f}{\partial\left(\partial_{Y Y} \Psi_{\mathrm{f}}\right)}\right)+\frac{\partial}{\partial x}\left(\frac{\partial f}{\partial\left(\partial_{x Y} \Psi_{\mathrm{f}}\right)}\right)=\frac{\partial}{\partial Y}\left(\frac{\partial f}{\partial\left(\partial_{Y Y} \Psi_{\mathrm{s}}\right)}\right)+\frac{\partial}{\partial x}\left(\frac{\partial f}{\partial\left(\partial_{x Y} \Psi_{\mathrm{s}}\right)}\right) \\
& \gamma^{2} \frac{\partial f}{\partial\left(\partial_{Y Y} \Psi_{\mathrm{f}}\right)}=\frac{\partial f}{\partial\left(\partial_{Y Y} \Psi_{\mathrm{s}}\right)} \\
& \frac{\partial}{\partial Y}\left(\frac{\partial f}{\partial\left(\partial_{Y Y} \Psi_{\mathrm{f}}\right)}\right)+\frac{\partial}{\partial x}\left(\frac{\partial f}{\partial\left(\partial_{x Y} \Psi_{\mathrm{f}}\right)}\right)=0 \\
& \frac{\partial f}{\partial\left(\partial_{Y Y} \Psi_{\mathrm{f}}\right)}=0 \\
& \lim _{Y \rightarrow-\infty} \partial_{x} \Psi_{\mathrm{s}}-Y=0 \\
& \lim _{Y \rightarrow-\infty} \partial_{Y} \Psi_{\mathrm{s}}-x=0 .
\end{aligned}
$$

The derivation of these conditions is given in [1].

\section{Appendix B. Comparison of amplitude of numerical and analytical solutions}

In order to compare the observed amplitude 4.6 with our analytical prediction, we recall that the vertical displacement can be computed from the stream function in the growth case by

$$
v=y-Y=\frac{\partial \Psi}{\partial x}-Y .
$$

Taking the first two terms of the perturbation expansion (3.1) and substituting in $(3.3)$, we find that

$$
\left.v\right|_{Y=1}=\gamma^{2}-1+\epsilon\left(i k a(\epsilon x, \epsilon t) h^{(1,1)}(1)+\text { c.c. }\right)+O\left(\epsilon^{2}\right) .
$$


Substituting in the constant amplitude solutions $(3.22)$, we then have

$$
\begin{aligned}
a_{\mathrm{obs}} & =\|v\|_{L^{\infty}(\{Y=1\} ; \mathbb{R})}-\left(\gamma^{2}-1\right) \\
& =2 k \sqrt{\frac{\mathscr{C}_{0}\left(\gamma-\gamma_{\mathrm{cr}}\right)}{\mathscr{C}_{1} \gamma_{\mathrm{cr}}}}\left|h_{\mathrm{f}}^{(1,1)}(1)\right|+O\left(\epsilon^{2}\right) .
\end{aligned}
$$

which can be computed explicitly to produce figures such as Figure 7.

[1] Alawiye, H., Kuhl, E., Goriely, A., 2019. Revisiting the wrinkling of elastic bilayers I: Linear analysis. Philosophical Transactions of the Royal Society A 377, 20180076.

[2] Amestoy, P. R., Duff, I. S., L'Excellent, J.-Y., Koster, J., 2001. A fully asynchronous multifrontal solver using distributed scheduling. SIAM Journal on Matrix Analysis and Applications 23 (1), $15-41$.

[3] Arnold, D. N., Qin, J., 1992. Quadratic velocity/linear pressure Stokes elements. In: Advances in Computer Methods for Partial Differential Equations VII. IMACS, pp. 28-34.

[4] Auricchio, F., Beirão da Veiga, L., Lovadina, C., Reali, A., Taylor, R. L., Wriggers, P., 2013. Approximation of incompressible large deformation elastic problems: some unresolved issues. Computational Mechanics 52, 1153-1167.

[5] Balay, S., Abhyankar, S., Adams, M. F., Brown, J., Brune, P., Buschelman, K., Dalcin, L., Dener, A., Eijkhout, V., Gropp, W. D., Karpeyev, D., Kaushik, D., Knepley, M. G., May, D. A., McInnes, L. C., Mills, R. T., Munson, T., Rupp, K., Sanan, P., Smith, B. F., Zampini, S., Zhang, H., Zhang, H., 2019. PETSc users manual. Tech. Rep. ANL-95/11 - Revision 3.11, Argonne National Laboratory.

URL http://www.mcs.anl.gov/petsc

[6] Ben Amar, M., Bordner, A., 2017. Mimicking cortex convolutions through the wrinkling of growing soft bilayers. Journal of Elasticity 129 (1-2), 213-238.

[7] Ben Amar, M., Jia, F., 2013. Anisotropic growth shapes intestinal tissues during embryogenesis. Proceedings of the National Academy of Sciences of the United States of America 110 (26), 10525-10530.

[8] Biot, M. A., 1963. Surface instability of rubber in compression. Applied Scientific Research, Section A 12 (2), 168-182.

[9] Bowden, N., Brittain, S., Evans, A. G., Hutchinson, J. W., Whitesides, G. M., 1998. Spontaneous formation of ordered structures in thin films of metals supported on an elastomeric polymer. Nature 393, 146-149.

[10] Braess, D., Ming, P., 2005. A finite element method for nearly incompressible elasticity problems. Mathematics of Computation 74 (249), 25-52.

[11] Brown, K. M., Gearhart, W. B., 1971. Deflation techniques for the calculation of further solutions of a nonlinear system. Numerische Mathematik 16 (4), 334-342.

[12] Budday, S., Kuhl, E., Hutchinson, J. W., 2015. Period-doubling and period-tripling in growing bilayered systems. Philos Mag 95 (28-30), 3208-3224.

[13] Cai, Z. X., Fu, Y. B., 1999. On the imperfection sensitivity of a coated elastic half-space. Proceedings of the Royal Society of London A, 3285-3309. 
[14] Cao, C., Chan, H. F., Zang, J., Leong, K. W., Zhao, X., 2014. Harnessing localized ridges for high-aspect-ratio hierarchical patterns with dynamic tunability and multifunctionality. Advanced Materials 26, 1763-1770.

[15] Cao, Y. P., Hutchinson, J. W., 2012. Wrinkling phenomena in neo-Hookean film/substrate bilayers. Journal of Applied Mechanics 79 (3).

[16] Cheewaruangroj, N., Biggins, J. S., 2019. Pattern selection when a layer buckles on a soft substrate. Soft Matter 15, 3751-3770.

[17] Chung, J. Y., Nolte, A. J., Stafford, C. M., 2011. Surface wrinkling: A versatile platform for measuring thin-film properties. Advanced Materials 23, 349-368.

[18] Ciarletta, P., 2018. Matched asymptotic solution for crease nucleation in soft solids. Nature Communications 9.

[19] Ciarletta, P., Balbi, V., Kuhl, E., 2014. Pattern selection in growing tubular tissues. Physical Review Letters 113 (24), 248101.

[20] Ciarletta, P., Ben Amar, M., 2012. Papillary networks in the dermal-epidermal junction of skin: a biomechanical model. Mechanics Research Communications 42, 68-76.

[21] Ciarletta, P., Fu, Y. B., 2015. A semi-analytical approach to Biot instability in a growing layer: Strain gradient correction, weakly non-linear analysis and imperfection sensitivity. International Journal of Non-Linear Mechanics 75, 38-45.

[22] Ciarletta, P., Truskinovsky, L., 2019. Soft nucleation of an elastic crease. Physical Review Letters $122,248001$.

[23] Diab, M., Kim, K.-S., 2014. Ruga-formation instabilities of a graded stiffness boundary layer in a neo-Hookean solid. Proceedings of the Royal Society A 470, 20140218.

[24] Diab, M., Zhang, T., Zhao, R., Gao, H., Kim, K.-S., 2013. Ruga mechanics of creasing: from instantaneous to setback creases. Proceedings of the Royal Society A 469, 20120753.

[25] Farrell, P. E., Birkisson, Á., Funke, S. W., 2015. Deflation techniques for finding distinct solutions of nonlinear partial differential equations. SIAM Journal on Scientific Computing 37 (4), A2026A2045.

[26] Fu, Y. B., Cai, Z. X., 2015. An asymptotic analysis of the period-doubling secondary bifurcation in a film/substrate bilayer. SIAM Journal on Applied Mathematics 75 (6), 2381-2395.

[27] Fu, Y. B., Cai, Z. X., 2015. An asymptotic analysis of the period-doubling secondary bifurcation in a film/substrate bilayer. SIAM J. Appl. Math. 75 (6), 2381-2395.

[28] Fu, Y. B., Ciarletta, P., 2015. Buckling of a coated elastic half-space when the coating and substrate have similar material properties. Proceedings of the Royal Society of London A 471 (2178).

[29] Fu, Y. B., Ogden, R. W., 1999. Nonlinear stability analysis of pre-stressed elastic bodies. Continuum Mechanics and Thermodynamics 11, 141-172.

[30] Goriely, A., 2017. The Mathematics and Mechanics of Biological Growth. Vol. 45 of Interdisciplinary Applied Mathematics. Springer-Verlag New York. 
[31] Goriely, A., Geers, M. G. D., Holzapfel, G. A., Jayamohan, J., Jérusalem, A., Sivaloganathan, S., Squier, W., van Dommelen, J. A. W., Waters, S., Kuhl, E., 2015. Mechanics of the brain: perspectives, challenges and opportunities. Biomechanics and Modeling in Mechanobiology 14 (5), 931-965.

[32] Goriely, A., Nizette, M., Tabor, M., 2001. On the dynamics of elastic strips. Journal of Nonlinear Science 11, 3-45.

[33] Hernandez, V., Roman, J. E., Vidal, V., 2005. SLEPc: A scalable and flexible toolkit for the solution of eigenvalue problems. ACM Transactions on Mathematical Software 31 (3), 351-362.

[34] Hohlfeld, E., 2013. Coexistence of scale-invariant states in incompressible elastomers. Physical Review Letters 111, 185701.

[35] Hohlfeld, E., Mahadevan, L., 2011. Unfolding the sulcus. Physical Review Letters 106 (10).

[36] Holland, M. A., Li, B., Feng, X. Q., Kuhl, E., 2017. Instabilities of soft films on compliant substrates. Journal of the Mechanics and Physics of Solids 98, 350-365.

[37] Hong, W., Zhao, X., Suo, Z., 2009. Formation of creases on the surfaces of elastomers and gels. Applied Physics Letters 95 (11).

[38] Hutchinson, J. W., 2013. The role of nonlinear substrate elasticity in the wrinkling of thin films. Philosophical Transactions of the Royal Society A 371, 20120422.

[39] Jin, L., Auguste, A., Hayward, R. C., Suo, Z., 2015. Bifurcation diagrams for the formation of wrinkles or creases in soft bilayers. Journal of Applied Mechanics 82 (6).

[40] Khang, D.-Y., Jiang, H., Huang, Y., Rogers, J. A., 2006. A stretchable form of single-crystal silicon for high-performance electronics on rubber substrates. Science 311 (5758), 208-212.

[41] Kim, J., Yoon, J., Hayward, R. C., 2010. Dynamic display of biomolecular patterns through an elastic creasing instability of stimuli-responsive hydrogels. Nature Materials 9, 159-164.

[42] Lacour, S. P., Jones, J., Suo, Z., Wagner, S., 2004. Design and performance of thin metal film interconnects for skin-like electronic circuits. IEEE Electron Device Letters 25 (4), 179-181.

[43] Lange, C. G., Newell, A. C., 1971. The post-buckling problem for thin elastic shells. SIAM Journal on Applied Mathematics 21 (4), 605-629.

[44] Lega, J., Goriely, A., 1999. Pulses, fronts and oscillations of an elastic rod. Physica D 132, 373391.

[45] Li, B., Cao., Y.-P., Feng., X.-Q., Gao, H., 2011. Surface wrinkling of mucosa induced by volumetric growth: theory, simulation and experiment. Journal of the Mechanics and Physics of Solids 59 (4), $758-774$.

[46] Li, B., Cao, Y.-P., Feng, X.-Q., Gao, H., 2012. Mechanics of morphological instabilities and surface wrinkling in soft materials: a review. Soft Matter $8(21), 5728-5745$.

[47] Moore, G., Spence, A., 1980. The calculation of turning points of nonlinear equations. SIAM Journal on Numerical Analysis 17 (4), 567-576.

[48] Olshanskii, M. A., Rebholz, L. G., 2011. Application of barycenter refined meshes in linear elasticity and incompressible fluid dynamics. Electronic Transactions on Numerical Analysis 38, 258274. 
[49] Rathgeber, F., Ham, D. A., Mitchell, L., Lange, M., Luporini, F., Mcrae, A. T. T., Bercea, G.-T., Markall, G. R., Kelly, P. H. J., 2016. Firedrake: automating the finite element method by composing abstractions. ACM Transactions on Mathematical Software 43 (3), 24:1-24:27.

[50] Stewart, G. W., 2001. A Krylov-Schur algorithm for large eigenproblems. SIAM Journal on Matrix Analysis and Applications 23 (3), 601-614.

[51] Tang, S., Gao, B., Zhou, Z., Gu, Q., Guo, T., 2017. Dimension-controlled formation of crease patterns on soft solids. Soft Matter 13, 619-626.

[52] Trujillo, V., Kim, J., Hayward, R. C., 2008. Creasing instability of surface-attached hydrogels. Soft Matter 4 (3), 564-569.

[53] Wang, Q., Zhao, X., 2015. A three-dimensional phase diagram of growth-induced surface instabilities. Scientific Reports 5.

[54] Whittaker, E. T., Watson, G. N., 1920. A Course of Modern Analysis. Cambridge University Press.

[55] Wong, W. H., Guo, T. F., Zhang, Y. W., Cheng, L., 2010. Surface instability maps for soft materials. Soft Matter 6, 5743-5750. 